\title{
Optimality of Impulse Control Problem in Refracted Lévy Model with Parisian Ruin and Transaction Costs
}

\author{
Irmina Czarna ${ }^{1}$. Adam Kaszubowski \\ Received: 8 July 2019 / Accepted: 1 May 2020 / Published online: 25 May 2020 \\ (c) The Author(s) 2020
}

\begin{abstract}
Here, we investigate an optimal dividend problem with transaction costs, in which the surplus process is modeled by a refracted Lévy process and the ruin time is considered with Parisian delay. The presence of the transaction costs implies that the impulse control problem needs to be considered as a control strategy in such a model. An impulse policy which involves reducing the reserves to some fixed level, whenever they are above another, is an important strategy for the impulse control problem. Therefore, we provide sufficient conditions under which the above described impulse policy is optimal. Furthermore, we provide new analytical formulae for the Parisian refracted $q$-scale functions in the case of the linear Brownian motion and the CrámerLundberg process with exponential claims. Using these formulae, we show that, for these models, there exists a unique policy, which is optimal for the impulse control problem. Numerical examples are also provided.
\end{abstract}

Keywords Refracted Lévy process · Parisian ruin · Dividend problem · Impulse control

Mathematics Subject Classification 60G40 · 60G51 · 93E20

Communicated by Nizar Touzi.

Irmina Czarna

irmina.czarna@pwr.edu.pl

Adam Kaszubowski

adam.kaszubowski@math.uni.wroc.pl

1 Faculty of Pure and Applied Mathematics, Wrocław University of Science and Technology, Wrocław, Poland

2 Mathematical Insititute, University of Wrocław, Wrocław, Poland 


\section{Introduction}

For many years, applied mathematicians have been trying to create models that allow describing reality in terms of mathematics. A special role is played by models used to describe phenomena that develop over time and exhibit some factor of randomness. In such a case, it is important to approximate certain characteristics or to find some event probabilities. For example, insurance companies need to estimate the amount of reserves that will allow them to be solvent with a very high probability. In this case, a question regarding the size of these reserves arises. Another example can be hedging companies, which when valuing financial instruments often use stochastic models.

In this study, we focus on another classic problem affecting companies called the issue of the optimal dividend payments. Dividend is the transfer of a certain portion of the company's finances to the investors, so it is one of the tools for shareholders to receive profits from the company's support. Dividends also may attract new investors to the company and thus provide further financing. In contrast, significant dividend payments result in a reduction in the company funds, thus leading to a significant increase in the probability of losing liquidity. Therefore, dividend payments must be made in an optimal manner, and they may be made up to the company's bankruptcy. The problem of bankruptcy is related to the ruin theory, traditionally considered in the context of insurance companies, where the ruin moment is related to the process of financial surplus, and in particular, with the surplus size at a given moment. The moment of ruin is defined classically as the first moment when the surplus process goes below the level of zero. Nowadays, such a moment of ruin has a small chance of occurrence because such companies control (and are controlled) so that the probability of such event stays at a very low level, whether through the impact of additional cash or prior to fixing of financial reserves. The probability of the ruin as well as the theory of ruin therefore plays a significant role. It is a determinant of the financial situation of the company, which allows the management to make strategic decisions. Therefore, it is important to investigate different definitions of the ruin and choose the proper one for our case.

The classical definition of ruin seems to be an intuitively obvious one, and if such a moment occurs, we expect that the company will immediately declare bankruptcy. In practice, very often, the investors or the government try to save the company from bankruptcy. Additionally, the too restrictive definition of the ruin as an economic determinant causes freezing of too much cash being secured, so the company grows weaker. Analyzing this problem in terms of the above doubts can lead to the conclusion that there is a natural need for a different definition of the ruin and in particular the separation of the technical ruin, i.e., exceeding the zero level, and the actual moment of bankruptcy announcement. Therefore, many alternatives have appeared in the literature, for example, the so-called Parisian ruin model, which is considered in this study. In this approach, we say that the company announces bankruptcy if the risk process goes below zero (or the so-called red zone) and stays there longer than a certain fixed time $r>0$. Such Parisian stopping times have been studied by Chesney et al. [1] in the context of barrier options in mathematical finance. In another paper, Czarna and Palmowski [2] gave the first description of the Parisian ruin probability for a general spectrally negative Lévy process. 
Now, let us define a class of the processes that are usually used to model the financial surplus. One of the most known stochastic processes used in the theory of ruin is the Crámer-Lundberg process. The form of this process has some benefits in the aspect of ease of calculations; however, sometimes it may turn out to be too far-reaching simplification. For example, one can see that between successive claims, this process is deterministic, so it does not consider certain market fluctuations. In addition, in the form of this process, we cannot distinguish between large and small claims, which is done in practice for insurance companies. Therefore, one can consider a wider class of spectrally negative Lévy processes that contain the Crámer-Lundberg process. This class of the processes includes linear Brownian motion, Cauchy process, and $\alpha$-stable processes.

As was mentioned before, one would like to distinguish the moment of exceeding the zero level with the actual bankruptcy by considering the Parisian ruin time. However, to further approximate the model with respect to reality, we will add an additional assumption. When the surplus process is in the red zone (i.e., below zero), we assume that it will receive a steady flow of cash with the intensity of $\delta>0$, until it will reach positive values. It reflects saving the company from bankruptcy by investors or the government. For such an assumption to be added, the so-called spectrally negative refracted Lévy process needs to be used, which was introduced by Kyprianou and Loeffen [3].

Therefore, using the above-mentioned assumptions, our goal is to analyze the problem of the optimal dividend payments, where each payment is to be accompanied by a certain fixed transaction fee $\beta>0$.

Historically, many studies have been published on this topic. The first problem was examined by de Finetti in [4]. He postulated that if the risk process behaves like a random walk with the increments of \pm 1 , then the optimal dividend strategy is of the barrier type. The barrier strategy is that the company pays everything above a certain fixed level $b$. The next step was to consider the continuous-type processes. In the framework of the linear Brownian motion process as well as the CramerLundberg process, a similar result was obtained, i.e., the optimal strategy is the barrier strategy (see [5-7]). Finally, in [8], the optimal barrier strategy for the entire class of spectrally negative Lévy processes was examined. The authors received certain conditions that would ensure that this strategy is the optimal one. Moreover, they expressed these conditions in the language of the so-called scale functions, which will be introduced in Sect. 2.3. Note that in the all above-mentioned studies, there were no transaction costs. The only exception is [9], where this assumption was made also for the class of the spectrally negative Lévy processes. Because of this assumption, further consideration of the barrier strategy was not possible; thus, it was replaced by an impulse control strategy, which will be described in detail in the following chapters. Moreover, in Sect. 3.2, sufficient conditions were obtained by providing the optimality of this dividend strategy.

The rest of this study is organized as follows. First, we introduce some basic notation and definitions related to the spectrally negative Lévy processes and the refracted counterpart. In particular, we introduce the scale functions and explain why they play a key role in this theory. In Sect. 2.2, we will describe the dividend problem and explain what we mean by the dividend strategy is the optimal strategy. Section 3 is the 
main part of this paper. We will introduce the impulse $\left(c_{1}, c_{2}\right)$ policy and will provide sufficient conditions that the derivative of the Parisian refracted scale is fulfilled to ensure that the strategy is optimal. The last part of this paper is an example section, where we will give new analytical formulae for the Parisian refracted scale functions in the case of the linear Brownian motion and the Crámer-Lundberg process with exponential claims. Using these formulae, we will show that for these models, there exists a unique impulse policy which is optimal for the impulse control problem. Numerical examples will also be provided.

\section{Mathematical Model}

\subsection{Surplus Process}

Let $\left(\Omega, \mathcal{F}, \mathbb{F}=\left\{\mathcal{F}_{t}: t \geq 0\right\}, \mathbb{P}\right)$ be the probability space that satisfies the usual conditions. On this probability space, we consider process $X=\left\{X_{t}\right\}_{t \geq 0}$ to be a spectrally negative Lévy process, namely the stochastic process issued from the origin that has stationary and independent increments and càdlàg paths that have no positive jump discontinuities. To avoid degenerate cases, we exclude the case where $X$ has monotone paths. As a strong Markov process, we shall endow $X$ with probabilities $\left\{\mathbb{P}_{x}: x \in \mathbb{R}\right\}$ such that under $\mathbb{P}_{x}$, we have $X_{0}=x$ with probability one. Furthermore, $\mathbb{E}_{x}$ denotes the expectation with respect to $\mathbb{P}_{x}$. Recall that $\mathbb{P}=\mathbb{P}_{0}$ and $\mathbb{E}=\mathbb{E}_{0}$. Every spectrally negative Lévy process can be represented by the triple $(\gamma, \sigma, \Pi)$ where $\gamma \in \mathbb{R}, \sigma \geq 0$ and $\Pi$ is a measure of $]-\infty, 0\left[\right.$, which satisfies $\int_{]-\infty, 0[}\left(1 \wedge x^{2}\right) \Pi(\mathrm{d} x)<$ $\infty$. The Laplace exponent of $X$ is defined by

$\psi(\theta):=\log \left(\mathbb{E}\left[e^{\theta X_{1}}\right]\right)=\gamma \theta+\frac{1}{2} \sigma^{2} \theta^{2}+\int_{]-\infty, 0[}\left(e^{\theta x}-1-\theta x \mathbf{1}_{\{-1<x<0\}}\right) \Pi(\mathrm{d} x)$,

for any $\theta \geq 0$. For background on spectrally negative Lévy processes, we refer the reader to $[10,11]$.

We assume that, in our model, the surplus process $R$ is modelled by spectrally negative refracted Lévy process, which means that we allow injecting (in continuous way) certain amount of money with intensity $\delta>0$ when reserves are below zero. Namely, one can define such a process as a uniquely strong solution $R=\left\{R_{t}\right\}_{t \geq 0}$ to the stochastic differential equation $d R_{t}=d X_{t}-\delta \mathbf{1}_{\left\{R_{t}>b\right\}} d t$, for $\delta>0$ and $b=0$. Note that, refracted process $R$ with $b \geq 0$ was examined before by Kyprianou and Loeffen in [3]. As in [12], we focus here on the case when the refraction level $b$ equals zero. Moreover, to be compatible with [3] and [12], we subtract $\delta$ on the positive half-line instead of adding it on the negative half-line; however, the practical effect is the same. From this equation, it is easy to observe that above the level $b$, process $R$ evolves as process $Y_{t}=X_{t}-\delta t$. Because the process $Y$ is a spectrally negative Lévy process with the Lévy triplet $(\gamma-\delta, \sigma, \Pi)$, its Laplace exponent is given by $\psi_{Y}(\theta)=\psi(\theta)-\delta \theta$. In particular, process $Y$ retains the probabilistic properties of the process $X$, e.g. the bounded/unbounded variation of the paths. Moreover, we want to emphasize here that process $R$ is no longer spatial homogeneous which means that it is not a Lévy process. 
In Section 3.2, we will prove that process $R$ is a Feller process, and we will present the form of its infinitesimal generator.

\subsection{Dividend Problem}

Let us now formally introduce the problem studied in this paper; in particular, we define the optimization criterion and then define the candidate for the optimal strategy. Denote $\pi$ as a dividend or control strategy, where $\pi=\left\{L_{t}^{\pi}\right\}_{t \geq 0}$ is a non-decreasing, left-continuous $\mathbb{F}$-adapted process that starts at zero. We will assume that process $L^{\pi}$ is a pure jump process, i.e.,

$$
L_{t}^{\pi}=\sum_{0 \leq s<t} \Delta L_{s}^{\pi}, \quad \text { for all } t \geq 0
$$

Here, by $\Delta L_{s}^{\pi}=L_{s+}^{\pi}-L_{s}^{\pi}$, we mean the jump of the process $L^{\pi}$ at time s. Therefore, random variable $L_{t}^{\pi}$ can be interpreted as a cumulated dividend to the time $t$. Note that, pure jump assumption is taken directly from the presence of nonzero transaction costs, and such control strategies as (1) are known as impulse controls. Let us define the controlled risk process $U^{\pi}=\left\{U_{t}^{\pi}\right\}_{t \geq 0}$ by the dividend strategy $\pi$ as $U_{t}^{\pi}:=R_{t}-L_{t}^{\pi}$. The company pays dividends up to its bankruptcy moment that is the Parisian ruin time in our model. Let us formally define it as $\kappa^{r}:=\inf \left\{t>0: t-\sup \left\{s<t: U_{s}^{\pi} \geq 0\right\} \geq r, U_{t}^{\pi}<0\right\}$, where $r>0$ is the so-called Parisian delay. Denote the value function of a dividend strategy $\pi$ as $v_{\pi}^{\kappa^{r}}(x)=\mathbb{E}_{x}\left[\int_{0}^{\kappa^{r}} e^{-q t} d\left(L_{t}^{\pi}-\sum_{0 \leq s<t} \beta \mathbf{1}_{\left\{\Delta L_{s}^{\pi}>0\right\}}\right)\right]$, for $x \geq 0$, where $q>0$ is the discount rate and $\beta>0$ denotes the transaction cost that occurs whenever the company pays dividends. With the assumption (1), the above integral can be interpreted as the following sum $v_{\pi}^{\kappa^{r}}(x)=\mathbb{E}_{x}\left[\sum_{0 \leq t<\kappa^{r}} e^{-q t}\left(\Delta L_{t}^{\pi}-\beta \mathbf{1}_{\left\{\Delta L_{t}^{\pi}>0\right\}}\right)\right]$, where $x \geq 0$. We call a strategy $\pi$ admissible if we do not get to the red zone due to dividend payments, i.e.,

$$
U_{t}^{\pi}-\Delta L_{t}^{\pi} \geq 0, \quad \text { for } t<\kappa^{r} .
$$

Let $\mathcal{A}$ be the set of all admissible dividend strategies. Our main goal is to find the optimal value function $v_{*}$ given by $v_{*}(x):=\sup _{\pi \in \mathcal{A}} v_{\pi}^{\kappa^{r}}(x)$ and the optimal strategy $\pi_{*} \in \mathcal{A}$, such that $v_{\pi_{*}}^{\kappa^{r}}(x)=v_{*}(x)$, for all $x \geq 0$.

\subsection{Exit Problems and Scale Functions}

In this section, we introduce key tools that will allow the optimality of the dividend strategy to be investigated. From viewpoint of application, one of the most important issues studied in the theory of Lévy processes is the so-called exit problem. The classical de Finetti dividend problem can also be expressed using exit identities; therefore, we will recall the basic results from this topic. First, for $a \in \mathbb{R}$, we define the following first-passage stopping times 


$$
\begin{array}{lll}
\tau_{a}^{-}=\inf \left\{t>0: X_{t}<a\right\} & \text { and } & \tau_{a}^{+}=\inf \left\{t>0: X_{t} \geq a\right\}, \\
\nu_{a}^{-}=\inf \left\{t>0: Y_{t}<a\right\} & \text { and } & v_{a}^{+}=\inf \left\{t>0: Y_{t} \geq a\right\}, \\
\kappa_{a}^{-}=\inf \left\{t>0: R_{t}<a\right\} & \text { and } & \kappa_{a}^{+}=\inf \left\{t>0: R_{t} \geq a\right\} .
\end{array}
$$

One can be interested in obtaining an semi-analytical representation of the expression (the so-called two-sided exit problem) $\mathbb{E}_{x}\left[e^{-q \tau_{c}^{+}} \mathbf{1}_{\left\{\tau_{c}^{+}<\tau_{0}^{-}\right\}}\right]$. That is, one would like to examine a unit payment made when the level $c$ is reached before the first moment when the level zero is exceeded. This payment is additionally discounted by a discount factor $q>0$. To obtain the analytical expression for the above expectation, let us define the following function.

For each $q \geq 0$, there exists a function $W^{(q)}: \mathbb{R} \rightarrow[0, \infty[$, called the $q$-scale function, that satisfies $W^{(q)}(x)=0$ for $x<0$ and is characterized on $[0, \infty$ [ as a strictly increasing and continuous function whose Laplace transform is given by

$$
\int_{0}^{\infty} e^{-\theta x} W^{(q)}(x) \mathrm{d} x=\frac{1}{\psi(\theta)-q}
$$

where $\Phi(q)=\sup \{\theta \geq 0: \psi(\theta)=q\}$ is the right-inverse of $\psi$ and $\theta>\Phi(q)$. We define the second scale function by $Z^{(q)}(x):=1+q \int_{0}^{x} W^{(q)}(y) d y$, for $x \in \mathbb{R}$. It turns out that for $-\infty<a \leq x \leq c<\infty$ and $q \geq 0$ (see e.g., [11])

$$
\mathbb{E}_{x}\left[e^{-q \tau_{c}^{+}} \mathbf{1}_{\left\{\tau_{c}^{+}<\tau_{a}^{-}\right\}}\right]=\frac{W^{(q)}(x-a)}{W^{(q)}(c-a)}
$$

and also for $q>0 \mathbb{E}_{x}\left[e^{-q \tau_{a}^{-}} \mathbf{1}_{\left\{\tau_{a}^{-}<\tau_{c}^{+}\right\}}\right]=Z^{(q)}(x-a)-\frac{Z^{(q)}(c-a)}{W^{(q)}(c-a)} W^{(q)}(x-a)$. Analogously, we can define the scale functions for Lévy process $Y$, and we will use notation $\mathbb{W}^{(q)}$ and $\mathbb{Z}^{(q)}$ for the first and second scale functions for $Y$, respectively. Define the scale function for refracted process $R$ as follows. For $q \geq 0$ and $x, a \in \mathbb{R}$

$$
w^{(q)}(x ; a):=W^{(q)}(x-a)+\delta \int_{0}^{x} \mathbb{W}^{(q)}(x-y) W^{(q)^{\prime}}(y-a) d y .
$$

In particular, we write $w^{(q)}(\cdot):=w^{(q)}(\cdot ; 0)$ when $a=0$. One can see that the above definition differs from the definition of scale functions for $X$ and $Y$. However, in [3], it was proved that for $-\infty<a \leq x \leq c<\infty$ and $q \geq 0 \mathbb{E}_{x}\left[e^{-q \kappa_{c}^{+}} \mathbf{1}_{\left\{\kappa_{c}^{+}<\kappa_{a}^{-}\right\}}\right]=$ $\frac{w^{(q)}(x ; a)}{w^{(q)}(c ; a)}$. Therefore, one can see that for process $R$, function $w^{(q)}$ gives the same representation for the two-sided exit problem as the scale functions $W^{(q)}$ and $\mathbb{W}^{(q)}$.

In this paper, we additionally consider Parisian ruin time, and from [12], it is known that

$$
\mathbb{E}_{x}\left[e^{-q \kappa_{a}^{+}} \mathbf{1}_{\left\{\kappa_{a}^{+}<\kappa^{r}\right\}}\right]=\frac{V^{(q)}(x)}{V^{(q)}(a)},
$$


where $V^{(q)}(x)=\int_{0}^{\infty} w^{(q)}(x ;-z) \frac{z}{r} \mathbb{P}\left(X_{r} \in d z\right)$. Because scale functions occur in many fluctuation identities, the natural question is if it is possible to calculate them explicitly. The answer is that for some particular, like Brownian motion with drift or Cramér-Lundberg process with exponential jumps, the form of functions $W^{(q)}, w^{(0)}$, and $V^{(0)}$ can be obtained explicitly (see [10-14]).

\subsection{Properties of Scale Functions}

In this part, we will investigate properties of the scale functions, which will be crucial for further proofs in this paper. At the beginning, let us cover the behavior of the scale functions at zero. Recall that $(\gamma, \sigma, \Pi)$ is a Lévy triple of the process $X$ and set $p:=\gamma+\int_{0}^{1} x \Pi(\mathrm{d} x)$ when process $X$ is of bounded variation (this quantity then represents drift of the process). Then,

$$
W^{(q)}(0+)= \begin{cases}\frac{1}{p}, & \text { when } \mathrm{X} \text { has bounded variation paths } \\ 0, & \text { otherwise. }\end{cases}
$$

From (3), one can see that the initial value of $w^{(q)}$ equals $W^{(q)}(-a)$. In contrast, for $V^{(q)}$, one can find in $[12,15]$ that

$$
V^{(q)}(0)=\int_{0}^{\infty} W^{(q)}(z) \frac{z}{r} \mathbb{P}\left(X_{r} \in d z\right)=e^{q r} .
$$

$W^{(q)^{\prime}}(0+):=\lim _{x \downarrow 0} W^{(q) \prime}(x)$ equals (see, e.g., [11]) $\frac{2}{\sigma^{2}}$ when $\sigma>0, \frac{\Pi(0, \infty)+q}{p^{2}}$ when $\sigma=0$ and $\Pi(0, \infty)<\infty$, in the other cases it equals $+\infty$. Moreover, for $w^{(q)}$, the following proposition was proved in [16].

Proposition 2.1 In general, $w^{(q)}(\cdot ; a)$ is a.e. continuously differentiable and its derivative is equal to $w^{(q)^{\prime}}(x ; a)=W^{(q)^{\prime}}(x-a)$, for $x<0$ and $w^{(q)^{\prime}}(x ; a)=\left(1+\delta \mathbb{W}^{(q)}(0)\right) W^{(q)^{\prime}}(x-a)+\delta \int_{0}^{x} \mathbb{W}^{(q)^{\prime}}(x-y) W^{(q)^{\prime}}(y-a) d y$, for $x \geq 0$. In particular, if $X$ is of unbounded variation, then $w^{(q)}(\cdot ; a)$ is $C^{1}(] a, \infty[)$. In contrast, if we assume that $W^{(q)}(\cdot-a) \in C^{1}(] a, \infty[)$ for $X$ is of bounded variation, then $w^{(q)}(\cdot ; a)$ is also $C^{1}(] a, \infty[\backslash\{0\})$.

\section{Impulse Strategy with the Parisian Ruin}

Let us present the candidate to be an optimal strategy for the dividend problem described in Sect. 2.2. Formally, we define the so-called impulse strategy $\pi_{c_{1}, c_{2}}$. We set two constants $c_{1}$ and $c_{2}$ such that $c_{2}>c_{1}+\beta$ and $c_{1} \geq 0$. Next, fix $\left\{\tau_{k}^{c_{1}, c_{2}}, k=1,2, \ldots\right\}$ as a set of the stopping times, such that $\tau_{k}^{c_{1}, c_{2}}=\inf \left\{t>0: R_{t}>\left[\left(R_{0} \vee c_{2}\right)+\left(c_{2}-c_{1}\right)(k-1)\right]\right\}, \quad \mathrm{k}=1,2, \ldots$. The strategy $\pi_{c_{1}, c_{2}}=\left\{L_{t}^{c_{1}, c_{2}}: t \geq 0\right\}$ is defined as 


$$
L_{t}^{c_{1}, c_{2}}=\mathbf{1}_{\left\{\tau_{1}^{c_{1}, c_{2}}<t\right\}}\left(\left[R_{0} \vee c_{2}\right]-c_{1}\right)+\sum_{k=2}^{\infty} \mathbf{1}_{\left\{\tau_{k}^{c_{1}, c_{2}}<t\right\}}\left(c_{2}-c_{1}\right), \quad \text { for } t \geq 0
$$

Then, the controlled risk process is of the form $U_{t}^{c_{1}, c_{2}}=R_{t}-L_{t}^{c_{1}, c_{2}}$. Note that in the terms of $U_{t}^{c_{1}, c_{2}}$, we can write $\tau_{1}^{c_{1}, c_{2}}=\inf \left\{t>0: U_{t}^{c_{1}, c_{2}}>c_{2}\right\}$ and $\tau_{k}^{c_{1}, c_{2}}=\inf \{t>$ $\tau_{k-1}^{c_{1}, c_{2}}: U_{t}^{c_{1}, c_{2}}>c_{2}$ \} for $k \geq 2$.

Therefore, the impulse strategy is to reduce the risk process to $c_{1}$ whenever the process exceeds level $c_{2}$. It is assumed that the distance between $c_{1}$ and $c_{2}$ must be greater than $\beta$ because after paying the transaction costs, there must be something left for the shareholders. Additionally, the condition that $c_{1} \geq 0$ is a consequence of (2). Before we give necessary conditions for the $\left(c_{1}, c_{2}\right)$ strategy to be optimal, we need to consider the form of the value function as a helping tool for further investigations.

\subsection{Representation of the Value Function}

Proposition 3.1 The function $v_{c_{1}, c_{2}}^{\kappa^{r}}$ for the strategy $\pi_{c_{1}, c_{2}}$ with the ruin time $\kappa^{r}$ is of the form

$$
v_{c_{1}, c_{2}}^{\kappa^{r}}(x)= \begin{cases}\left(c_{2}-c_{1}-\beta\right) \frac{V^{(q)}(x)}{V^{(q)}\left(c_{2}\right)-V^{(q)}\left(c_{1}\right)}, & \text { for } x \leq c_{2}, \\ x-c_{1}-\beta+\left(c_{2}-c_{1}-\beta\right) \frac{V^{(q)}\left(c_{1}\right)}{V^{(q)}\left(c_{2}\right)-V^{(q)}\left(c_{1}\right)}, & \text { for } x>c_{2} .\end{cases}
$$

Proof At the beginning of the proof, note that it is sufficient to prove this Proposition only for $x \leq c_{2}$ because $U^{c_{1}, c_{2}}$ is a Markov process and if we are above level $c_{2}$, we put the process into level $c_{1}$ immediately. Assume that $x \leq c_{2}$.

The first time when we paid dividends is $\tau_{1}^{c_{1}, c_{2}}$, which means that we must wait until the first time when process $U^{c_{1}, c_{2}}$ is greater than $c_{2}$. Using a strong Markov property, we have

$$
v_{c_{1}, c_{2}}^{\kappa^{r}}(x)=\mathbb{E}_{x}\left[e^{-q \kappa_{c_{2}}^{+}} \mathbf{1}_{\left\{\kappa_{\left.c_{2}+<\kappa^{r}\right\}}\right.}\right] v_{c_{1}, c_{2}}^{\kappa^{r}}\left(c_{2}\right)=\frac{V^{(q)}(x)}{V^{(q)}\left(c_{2}\right)} v_{c_{1}, c_{2}}^{\kappa^{r}}\left(c_{2}\right),
$$

where the last equality follows from (4). If we are at point $c_{2}$, we paid $c_{2}-c_{1}-\beta$ and decrease $U^{c_{1}, c_{2}}$ by $c_{2}-c_{1}$. Again, by strong Markov property, we have

$$
v_{c_{1}, c_{2}}^{\kappa^{r}}\left(c_{2}\right)=c_{2}-c_{1}-\beta+v_{c_{1}, c_{2}}^{\kappa^{r}}\left(c_{1}\right)=c_{2}-c_{1}-\beta+\frac{V^{(q)}\left(c_{1}\right)}{V^{(q)}\left(c_{2}\right)} v_{c_{1}, c_{2}}^{\kappa^{r}}\left(c_{2}\right) .
$$

The next step is to solve the above equation with respect to $v_{c_{1}, c_{2}}^{\kappa^{r}}$. We obtain $v_{c_{1}, c_{2}}^{\kappa^{r}}\left(c_{2}\right)=\frac{V^{(q)}\left(c_{2}\right)}{V^{(q)}\left(c_{2}\right)-V^{(q)}\left(c_{1}\right)}\left(c_{2}-c_{1}-\beta\right)$. Finally, we must put the above formula into (8) to get the result. 
The idea of finding the optimal points $\left(c_{1}, c_{2}\right)$ leads to finding the minimum of the function below

$$
g\left(c_{1}, c_{2}\right)=\frac{V^{(q)}\left(c_{2}\right)-V^{(q)}\left(c_{1}\right)}{c_{2}-c_{1}-\beta} .
$$

Let us denote the domain of $g$ as $\operatorname{dom}(g)=\left\{\left(c_{1}, c_{2}\right): c_{1} \geq 0, c_{2}>c_{1}+\beta\right\}$. Let $C^{*}$ be a set of $\left(c_{1}, c_{2}\right)$ from $\operatorname{dom}(g)$ that minimizes function $\mathrm{g}$, namely $C^{*}=\left\{\left(c_{1}^{*}, c_{2}^{*}\right) \in \operatorname{dom}(g): \inf _{\left(c_{1}, c_{2}\right) \in \operatorname{dom}(g)} g\left(c_{1}, c_{2}\right)=g\left(c_{1}^{*}, c_{2}^{*}\right)\right\}$. Also fix set $\mathcal{B}=\left\{\left(c_{1}, c_{2}\right):\left(c_{1}, c_{2}\right) \in \operatorname{dom}(g), c_{1} \neq 0\right\}$.

Proposition 3.2 For $W^{(q)} \in C^{1}(0, \infty)$, the set $C^{*}$ is not empty and for each $\left(c_{1}^{*}, c_{2}^{*}\right) \in$ $C^{*}$, we have

$$
V^{(q) \prime}\left(c_{2}^{*}\right)=\frac{V^{(q)}\left(c_{2}^{*}\right)-V^{(q)}\left(c_{1}^{*}\right)}{c_{2}^{*}-c_{1}^{*}-\beta} .
$$

Also, we know that in this case there are the following possibilities:

(i) $V^{(q) \prime}\left(c_{1}^{*}\right)=V^{(q)^{\prime}}\left(c_{2}^{*}\right)$ or $(i i) c_{1}^{*}=0$.

Proof At the beginning, we will show that if $c_{1} \rightarrow \infty$ function $g$ is not attaining its minimum.

$$
\begin{aligned}
g\left(c_{1}, c_{2}\right) & =\int_{0}^{\infty}\left(\frac{w^{(q)}\left(c_{2} ;-z\right)-w^{(q)}\left(c_{1} ;-z\right)}{c_{2}-c_{1}-\beta}\right) \frac{z}{r} \mathbb{P}\left(X_{r} \in d z\right) \\
& \geq \int_{0}^{\infty}\left(\frac{W^{(q)}\left(c_{2}+z\right)-W^{(q)}\left(c_{1}+z\right)}{c_{2}-c_{1}}\right)\left(\frac{c_{2}-c_{1}}{c_{2}-c_{1}-\beta}\right) \frac{z}{r} \mathbb{P}\left(X_{r} \in d z\right) \\
& >\int_{0}^{\infty} \min _{x \in\left[c_{1}+z, c_{2}+z\right]} W^{(q) \prime}(x) \frac{z}{r} \mathbb{P}\left(X_{r} \in d z\right) \\
& \geq \int_{0}^{\infty} \min _{x \in\left[c_{1}, \infty[\right.} W^{(q) \prime}(x) \frac{z}{r} \mathbb{P}\left(X_{r} \in d z\right) \\
& =\min _{x \in\left[c_{1}, \infty[\right.} W^{(q) \prime}(x) \int_{0}^{\infty} \frac{z}{r} \mathbb{P}\left(X_{r} \in d z\right) \stackrel{c_{1} \rightarrow \infty}{\longrightarrow} \infty .
\end{aligned}
$$

In the first inequality, we used

$$
w^{(q)}\left(c_{2} ;-z\right)-w^{(q)}\left(c_{1} ;-z\right) \geq W^{(q)}\left(c_{2}+z\right)-W^{(q)}\left(c_{1}+z\right)
$$

The next inequality follows from the mean value theorem $\left(W^{(q)} \in C^{1}(] 0, \infty[)\right)$ and the simple fact that $\frac{c_{2}-c_{1}}{c_{2}-c_{1}-\beta}>1$. The last inequality is a consequence of $\left[c_{1}+z, c_{2}+z\right] \subseteq\left[c_{1}, \infty\left[\right.\right.$ for all $\left(c_{1}, c_{2}\right) \in \operatorname{dom}(g)$ and all $z>0$. Note that $\int_{0}^{\infty} \frac{z}{r} \mathbb{P}\left(X_{r} \in d z\right)>0$ and for that reason, the last statement follows. We get that $\inf _{\left(c_{1}, c_{2}\right) \in \operatorname{dom}(g)} g\left(c_{1}, c_{2}\right)$ is not attained when $c_{1} \rightarrow \infty$; thus, $\inf _{\left(c_{1}, c_{2}\right) \in \operatorname{dom}(g)} g\left(c_{1}, c_{2}\right)=\inf _{\left(c_{1}, c_{2}\right) \in \operatorname{dom}(g) \wedge c_{1} \leq C_{1}} g\left(c_{1}, c_{2}\right)$, for some $C_{1}>0$. In the next step, we will show the same for $c_{2}$. Namely 


$$
\begin{aligned}
\inf _{c_{1} \in\left[0, C_{1}\right]} g\left(c_{1}, c_{2}\right) \geq & \inf _{c_{1} \in\left[0, C_{1}\right]} \int_{0}^{\infty}\left(\frac{W^{(q)}\left(c_{2}+z\right)-W^{(q)}\left(c_{1}+z\right)}{c_{2}-c_{1}-\beta}\right) \frac{z}{r} \mathbb{P}\left(X_{r} \in d z\right) \\
\geq & \left(\frac{W^{(q)}\left(c_{2}\right)}{c_{2}-\beta} \int_{0}^{\infty} \frac{z}{r} \mathbb{P}\left(X_{r} \in d z\right)\right. \\
& \left.-\frac{1}{c_{2}-C_{1}-\beta} \int_{0}^{\infty} W^{(q)}\left(C_{1}+z\right) \frac{z}{r} \mathbb{P}\left(X_{r} \in d z\right)\right) \stackrel{c_{2} \rightarrow \infty}{\longrightarrow} \infty .
\end{aligned}
$$

Note that we used only (11) (in the first inequality) and the property that $W^{(q)}$ is increasing (in the second inequality). The last step is to consider the case when $\left(c_{1}, c_{2}\right)$ converges to the line $c_{2}=c_{1}+\beta$.

$$
\begin{aligned}
g\left(c_{1}, c_{2}\right) & =\int_{0}^{\infty}\left(\frac{w^{(q)}\left(c_{2} ;-z\right)-w^{(q)}\left(c_{1} ;-z\right)}{c_{2}-c_{1}-\beta}\right) \frac{z}{r} \mathbb{P}\left(X_{r} \in d z\right) \\
& \geq \int_{0}^{\infty} \min _{x \in\left[c_{1}+z, c_{2}+z\right]} W^{(q)^{\prime}}(x)\left(\frac{\beta}{c_{2}-c_{1}-\beta}\right) \frac{z}{r} \mathbb{P}\left(X_{r} \in d z\right) \\
& \geq W^{(q)^{\prime}}\left(a^{*}\right) \frac{\beta}{c_{2}-c_{1}-\beta} \int_{0}^{\infty} \frac{z}{r} \mathbb{P}\left(X_{r} \in d z\right) \rightarrow \infty .
\end{aligned}
$$

We used, again, the mean value theorem and fact that $c_{2}>c_{1}+\beta$. We check that infimum of $g$ is not reached when $c_{1} \rightarrow \infty$ or $c_{2} \rightarrow \infty$ or $\left(c_{1}, c_{2}\right)$ converges to $c_{2}=c_{1}+\beta$. Because of it and the continuity of $g$, we get that $C^{*}$ is not empty, and we are left with the following possibilities. First is that $\left(c_{1}^{*}, c_{2}^{*}\right)$ belongs to the interior of $\mathcal{B}$. In this case, using the fact that $g$ is partially differentiable in $c_{1}$ and $c_{2}$ $\left(W^{(q)} \in C^{1}(] 0, \infty[)\right)$, we get that $\frac{\partial g\left(c_{1}, c_{2}\right)}{\partial c_{1}}\left(c_{1}^{*}\right)=0$ and $\frac{\partial g\left(c_{1}, c_{2}\right)}{\partial c_{2}}\left(c_{2}^{*}\right)=0$. Hence, we obtain (10) and $(i)$. The second possibility is when $c_{1}^{*}=0$. Then, we have that $c_{2}^{*}$ minimizes function

$g_{0}\left(c_{2}\right)=g\left(0, c_{2}\right)=\frac{V^{(q)}\left(c_{2}\right)-V^{(q)}(0)}{c_{2}-\beta}$. We get $(i i)$ because $g_{0}^{\prime}\left(c_{2}^{*}\right)=0$.

To start the optimization reasoning, we need the following proposition and lemma.

Proposition 3.3 Assume that $W^{(q)} \in C^{1}(] 0, \infty[)$. For each $\left(c_{1}^{*}, c_{2}^{*}\right) \in C^{*}$, we have that $v_{c_{1}^{*}, c_{2}^{*}}^{\kappa^{r}}(x)=\frac{V^{(q)}(x)}{V^{(q) \prime}\left(c_{2}^{*}\right)}$, for $x \leq c_{2}^{*}$ and $v_{c_{1}^{*}, c_{2}^{*}}^{\kappa^{r}}(x)=\left(x-c_{2}^{*}\right)+\frac{V^{(q)}\left(c_{2}^{*}\right)}{V^{(q) \prime}\left(c_{2}^{*}\right)}$, for $x>c_{2}^{*}$.

Proof From Proposition (3.2), it follows that:

For $x \leq c_{2}^{*}, v_{c_{1}^{*}, c_{2}^{*}}^{\kappa^{r}}(x)=\left(c_{2}^{*}-c_{1}^{*}-\beta\right) \frac{V^{(q)}(x)}{V^{(q)}\left(c_{2}^{*}\right)-V^{(q)}\left(c_{1}^{*}\right)}=\frac{V^{(q)}(x)}{V^{(q)^{\prime}}\left(c_{2}^{*}\right)}$.

For $x>c_{2}^{*}$,

$$
\begin{aligned}
v_{c_{1}^{*}, c_{2}^{*}}^{\kappa^{r}}(x) & =x-c_{1}^{*}-\beta+\left(c_{2}^{*}-c_{1}^{*}-\beta\right) \frac{V^{(q)}\left(c_{1}^{*}\right)}{V^{(q)}\left(c_{2}^{*}\right)-V^{(q)}\left(c_{1}^{*}\right)} \\
& =x-c_{2}^{*}+\left(c_{2}^{*}-c_{1}^{*}-\beta\right) \frac{V^{(q)}\left(c_{2}^{*}\right)}{V^{(q)}\left(c_{2}^{*}\right)-V^{(q)}\left(c_{1}^{*}\right)} \\
& =x-c_{2}^{*}+\frac{V^{(q)}\left(c_{2}^{*}\right)}{V^{(q) \prime}\left(c_{2}^{*}\right)} .
\end{aligned}
$$


Lemma 3.1 Let $\left(c_{1}^{*}, c_{2}^{*}\right) \in C^{*}$ and $x \geq y \geq 0$. Then the following inequality $v_{c_{1}^{*}, c_{2}^{*}}^{\kappa^{r}}(x)-v_{c_{1}^{*}, c_{2}^{*}}^{\kappa^{r}}(y) \geq x-y-\beta$ holds.

Proof Note that $v_{c_{1}^{*}, c_{2}^{*}}^{\kappa^{r}}$ is an increasing function, and because of that, one can assume $x-y>\beta$. Consider the following possibilities. For $c_{2}^{*} \leq y \leq x$, one can obtain that $v_{c_{1}^{*}, c_{2}^{*}}^{\kappa^{r}}(x)-v_{c_{1}^{*}, c_{2}^{*}}^{\kappa^{r}}(y)=x-y>x-y-\beta$.

For $y \leq x \leq c_{2}^{*}$,

$$
\begin{aligned}
v_{c_{1}^{*}, c_{2}^{*}}^{\kappa^{r}}(x)-v_{c_{1}^{*}, c_{2}^{*}}^{\kappa^{r}}(y) & =\frac{\left(c_{2}^{*}-c_{1}^{*}-\beta\right)\left(V^{(q)}(x)-V^{(q)}(y)\right)}{V^{(q)}\left(c_{2}^{*}\right)-V^{(q)}\left(c_{1}^{*}\right)} \\
& \geq \frac{(x-y-\beta)\left(V^{(q)}(x)-V^{(q)}(y)\right)}{V^{(q)}(x)-V^{(q)}(y)}=x-y-\beta .
\end{aligned}
$$

The above inequality follows from fact that $\left(c_{1}^{*}, c_{2}^{*}\right) \in C^{*}$, so $\left(c_{1}^{*}, c_{2}^{*}\right)$ minimizes function $g\left(c_{1}, c_{2}\right)=\frac{V^{(q)}\left(c_{2}\right)-V^{(q)}\left(c_{1}\right)}{c_{2}-c_{1}-\beta}$.

For $y \leq c_{2}^{*} \leq x$,

$$
\begin{aligned}
v_{c_{1}^{*}, c_{2}^{*}}^{\kappa^{r}}(x)-v_{c_{1}^{*}, c_{2}^{*}}^{\kappa^{r}}(y) & =x-c_{1}^{*}-\beta+\left(c_{2}^{*}-c_{1}^{*}-\beta\right)\left(\frac{V^{(q)}\left(c_{1}^{*}\right)-V^{(q)}(y)}{V^{(q)}\left(c_{2}^{*}\right)-V^{(q)}\left(c_{1}^{*}\right)}\right) \\
& =x-c_{2}^{*}+\left(c_{2}^{*}-c_{1}^{*}-\beta\right)\left(1+\frac{V^{(q)}\left(c_{1}^{*}\right)-V^{(q)}(y)}{V^{(q)}\left(c_{2}^{*}\right)-V^{(q)}\left(c_{1}^{*}\right)}\right) \\
& =x-c_{2}^{*}+\left(c_{2}^{*}-c_{1}^{*}-\beta\right)\left(\frac{V^{(q)}\left(c_{2}^{*}\right)-V^{(q)}(y)}{V^{(q)}\left(c_{2}^{*}\right)-V^{(q)}\left(c_{1}^{*}\right)}\right) \\
& \geq x-y-\beta .
\end{aligned}
$$

The last inequality follows from point (ii) with $x=c_{2}^{*}$.

\subsection{Optimality}

For the remainder of the section, we will focus on verifying the optimality of the impulse strategy at the threshold level $\left(c_{1}^{*}, c_{2}^{*}\right)$. The proof is led by standard Markovian arguments to show that the impulse strategy fulfils the Verification Lemma. However, at the beginning, we will prove the following fact.

Fact 3.1 Refracted process $R$ is a Feller process and its infinitesimal generator is of the form

$$
\begin{aligned}
\Gamma f(x)= & \left(\gamma-\delta \mathbf{1}_{\{x>0\}}\right) f^{\prime}(x)+\frac{1}{2} \sigma^{2} f^{\prime \prime}(x) \\
& -\int_{0}^{\infty}\left(f(x+z)-f(x)-f^{\prime}(x) z \mathbf{1}_{\{0<z<1\}}\right) \Pi(d z),
\end{aligned}
$$


where $x \in \mathbb{R}$ and $f$ is a function on $\mathbb{R}$ such that $\Gamma f(x)$ is well defined.

Proof For $q>0, x \in \mathbb{R}$ and a nonnegative or bounded measurable function $f$ define $P_{R}^{(q)} f:=\mathbb{E}_{x}\left[\int_{0}^{\infty} e^{-q t} f\left(R_{t}\right) d t\right]$. It is sufficient to verify the following conditions:

1. For all $q, p>0, P_{R}^{(q)}-P_{R}^{(p)}=(p-q) P_{R}^{(q)} P_{R}^{(p)}$.

2. For all $q>0,\left\|q P_{R}^{(q)} 1\right\| \leq 1$.

3. For all $q>0, P_{R}^{(q)}$ is a map from $C_{0}$ to $C_{0}$.

4. For all $f \in C_{0}, \lim _{q \rightarrow \infty}\left\|q P_{R}^{(q)} f-f\right\|=0$.

Here, $C_{0}(\mathbb{R})$ denotes the space of continuous functions vanishing at infinity. It is a Banach space when equipped with the uniform norm $\|f\|=\sup _{x \in \mathbb{R}}|f(x)|$. Because the process $R$ is a Strong Markov process (for details see [3]), one can observe that condition (1) is automatically fulfilled. Condition (2) is obvious. To prove (3) and (4), the reasoning is similar as in [17] except that we need to use fluctuation identities obtained in [3]. The form of the generator follows, i.e., from [18] with $l(x)=\gamma-$ $\delta \mathbf{1}_{\{x>0\}}$ and $Q(x)=\sigma^{2}$.

Lemma 3.2 (Verification Lemma) Suppose $\hat{\pi}$ is an admissible dividend strategy such that $v_{\hat{\pi}}$ is sufficiently smooth on $\mathbb{R}$ (i.e., its first or second derivative (for $X$ of bounded or unbounded variations, respectively) has at most a finite number of single discontinuities), satisfies

$$
\begin{aligned}
& (\Gamma-q) v_{\hat{\pi}}(x) \leq 0, \text { for } x \in \mathbb{R}, \\
& v_{\hat{\pi}}(x)-v_{\hat{\pi}}(y) \geq x-y-\beta, \text { for } x \geq y .
\end{aligned}
$$

Then, $v_{\hat{\pi}}(x)=v_{*}(x)$ for almost every $x \in \mathbb{R}$ and hence $\hat{\pi}=\pi_{*}$ is an optimal strategy.

Proof By the definition of $v_{*}$ as a supremum, it follows that $v_{\hat{\pi}}(x) \leq v_{*}(x)$ for all $x \in \mathbb{R}$. We write $h:=v_{\hat{\pi}}$ and show that $h(x) \geq v_{\pi}(x)$ for all $\pi \in \mathcal{A}$ for all $x \in \mathbb{R}$.

Fix $\pi \in \mathcal{A}$. Let $\left(T_{n}\right)_{n \in \mathbb{N}}$ be the sequence of stopping times defined by $T_{n}:=\inf \{t>$ $0: U_{t}^{\pi}>n$ or $\left.t-\sup \left\{s \leq t: U_{t}^{\pi} \geq 1 / n\right\}>r\right\}$. Because $U^{\pi}$ is a semimartingale (see, e.g., [19], [20]) and $h$ is sufficiently smooth on ]0, $\infty$ [, we will use to the stopped process $\left(e^{-q\left(t \wedge T_{n}\right)} h\left(U_{t \wedge T_{n}}^{\pi}\right) ; t \geq 0\right)$ the Bouleau and Yor [21] formula for bounded variation processes and the change of variables/Meyer- Itô's formula (cf. Theorem IV.71 of [22]) for unbounded variation case, and deduce that under $\mathbb{P}_{x}$ :

$$
\begin{aligned}
e^{-q\left(t \wedge T_{n}\right)} h\left(U_{t \wedge T_{n}}^{\pi}\right)-h(x)= & -\int_{0}^{t \wedge T_{n}} e^{-q s} q h\left(U_{s-}^{\pi}\right) \mathrm{d} s \\
& +\frac{\sigma^{2}}{2} \int_{0}^{t \wedge T_{n}} e^{-q s} h^{\prime \prime}\left(U_{s-}^{\pi}\right) \mathrm{d} s \\
& +\int_{\left[0, t \wedge T_{n}\right]} e^{-q s} h^{\prime}\left(U_{s-}^{\pi}\right) \mathrm{d}\left(R_{S}-L_{s}^{\pi}\right) \\
& +\sum_{0 \leq s \leq t \wedge T_{n}} e^{-q s}\left[\Delta h\left(U_{s-}^{\pi}+\Delta R_{S}\right)-h^{\prime}\left(U_{s-}^{\pi}\right) \Delta R_{S}\right],
\end{aligned}
$$


where we use the following notation: $\Delta \zeta(s):=\zeta(s)-\zeta(s-)$ and $\Delta h(\zeta(s)):=h(\zeta(s))-h(\zeta(s-))$ for any process $\zeta$ with left-hand limits. Rewriting the above equation leads to

$$
\begin{aligned}
e^{-q\left(t \wedge T_{n}\right)} h\left(U_{t \wedge T_{n}}^{\pi}\right)-h(x)= & \int_{0}^{t \wedge T_{n}} e^{-q s}(\Gamma-q) h\left(U_{s-}^{\pi}\right) \mathrm{d} s \\
& -\int_{0}^{t \wedge T_{n}} e^{-q s} h^{\prime}\left(U_{s-}^{\pi}\right) \mathrm{d} L_{s}^{\pi}+M_{t \wedge T_{n}},
\end{aligned}
$$

where $\left\{M_{t}: t \geq 0\right\}$ is a zero-mean $\mathbb{P}_{x}$-martingale.

Hence, using the assumptions (13), (14) we obtain the following

$$
\begin{aligned}
h(x) \geq & \int_{0}^{t \wedge T_{n}} e^{-q s} \mathrm{~d}\left(L_{s}^{\pi}-\beta \sum_{0 \leq z \leq s} \mathbf{1}_{\left\{\Delta L_{z}^{\pi}>0\right\}}\right)-M_{t \wedge T_{n}} \\
& +e^{-q\left(t \wedge T_{n}\right)} h\left(U_{t \wedge T_{n}}^{\pi}\right) .
\end{aligned}
$$

Now, taking expectations in (15), using the fact that $\left(M_{t \wedge T_{n}}: t \geq 0\right)$ is a zero-mean $\mathbb{P}_{x}$-martingale and $h \geq 0$, letting $t$ and $n$ go to infinity $\left(T_{n} \stackrel{n \uparrow \infty}{\longrightarrow} \kappa^{r} \mathbb{P}_{x}\right.$-a.s.), the dominated convergence gives

$$
\begin{aligned}
h(x) \geq & \lim _{t, n \uparrow \infty} \mathbb{E}_{x}\left[\int_{0}^{t \wedge T_{n}} e^{-q s} \mathrm{~d}\left(L_{s}^{\pi}-\beta \sum_{0 \leq z \leq s} \mathbf{1}_{\left\{\Delta L_{z}^{\pi}>0\right\}}\right)-M_{t \wedge T_{n}}\right. \\
& \left.+e^{-q\left(t \wedge T_{n}\right)} h\left(U_{t \wedge T_{n}}^{\pi}\right)\right] \\
\geq & \mathbb{E}_{x}\left[\int_{0}^{\kappa^{r}} e^{-q s} \mathrm{~d}\left(L_{s}^{\pi}-\beta \sum_{0 \leq z \leq s} \mathbf{1}_{\left\{\Delta L_{z}^{\pi}>0\right\}}\right)+\lim _{t, n \uparrow \infty} e^{-q\left(t \wedge T_{n}\right)} h\left(U_{t \wedge T_{n}}^{\pi}\right)\right] \\
\geq & v_{\pi}(x),
\end{aligned}
$$

which completes the proof.

Remark 3.1 The lemma presented below requires some smoothness on the NPV of a $\left(c_{1} ; c_{2}\right)$ policy. In the view of Proposition 3.3, it means that some smoothness conditions on the scale function $V^{(q)}$ are required. We will call the scale function $V^{(q)}$ sufficiently smooth if $W^{(q)} \in C^{1}(] 0, \infty[)$ when $X$ is of bounded variation. From Theorem 2.9 of [23], one can see that a necessary and sufficient condition for this is that the Lévy measure has no atoms. When $\mathrm{X}$ is of unbounded variation, we call the scale function $V^{(q)}$ sufficiently smooth if $W^{(q)} \in C^{1}(] 0, \infty[)$ and $W^{(q)^{\prime}}$ is absolutely continuous on ]0, $\infty$ [ with a density which is bounded on sets of the form $[1 / n, n]$, $n \geq 1$. Moreover, in Theorem 2.6 of [23], it is proved that $W^{(q)} \in C^{2}(] 0, \infty[)$ if the Gaussian coefficient $\sigma$ is strictly positive. Note that the term sufficiently smooth is used here in a slightly weaker sense (which is explained in detail in the Lemma below). 
Lemma 3.3 If $V^{(q)}$ is sufficiently smooth and fulfils (13) and (14), then $v_{c_{1}^{*}, c_{2}^{*}}^{\kappa^{r}}=v_{*}$ for almost every $x \in \mathbb{R}$.

Proof From Lemma 3.1, one can see that it is sufficient to prove that (13) holds. At first to get that $(\Gamma-q) v_{c_{1}^{*}, c_{2}^{*}}^{\kappa^{r}}=0$, for $x<c_{2}^{*}$, one can observe that from Proposition 3.3 (for $x<c_{2}^{*}$ ), it is enough to show that $\left(e^{-q\left(t \wedge \kappa^{r} \wedge \kappa_{c}^{+}\right)} V^{(q)}\left(R_{t \wedge \kappa^{r} \wedge \kappa_{c}^{+}}\right)\right)_{t \geq 0}$ is a $\mathbb{P}_{x}$-martingale. Indeed, let $\tau:=\kappa^{r} \wedge \kappa_{c}^{+}$, using (4) together with the fact that $V^{(q)}\left(R_{\tau}\right) / V^{(q)}(c)=\mathbf{1}_{\left\{\kappa_{c}^{+}<\kappa^{r}\right\}}$, one can get

$$
\begin{aligned}
\mathbb{E}_{x}\left[e^{-q \tau} V^{(q)}\left(R_{\tau}\right) \mid \mathcal{F}_{t}\right] & =\mathbf{1}_{\{t \leq \tau\}} e^{-q t} \mathbb{E}_{R_{t}}\left[e^{-q \tau} V^{(q)}\left(R_{\tau}\right)\right]+\mathbf{1}_{\{\tau<t\}} e^{-q \tau} V^{(q)}\left(R_{\tau}\right) \\
& =\mathbf{1}_{\{t \leq \tau\}} e^{-q t} V^{(q)}\left(R_{\tau}\right)+\mathbf{1}_{\{\tau<t\}} e^{-q \tau} V^{(q)}\left(R_{\tau}\right) \\
& =e^{-q(t \wedge \tau)} V^{(q)}\left(R_{t \wedge \tau}\right) .
\end{aligned}
$$

From Proposition 2.1, the derivative of scale function $V^{(q)}$ does not exist at 0 , when $X$ is of bounded variation. Moreover, when $\sigma>0$, the second left derivative of $v_{c_{1}^{*}, c_{2}^{*}}^{\kappa^{r}}$ at $c_{2}^{*}$ does not equal zero, and hence, $(\Gamma-q) v_{c_{1}^{*}, c_{2}^{*}}^{\kappa^{r}}$ is not well defined. Therefore, we claim that the result below holds for almost every $x \in \mathbb{R}$. Indeed, it is sufficient to show that for any $t>0$

$$
\int_{0}^{t} e^{-q s}(\Gamma-q) v_{c_{1}^{*}, c_{2}^{*}}^{\kappa^{r}}\left(\tilde{U}_{s}^{c_{1}^{*}, c_{2}^{*}}\right) d s \leq 0
$$

almost surely, where $\tilde{U}^{c_{1}^{*}, c_{2}^{*}}$ is the right-continuous modification of $U^{c_{1}^{*}, c_{2}^{*}}$. One can prove it using the occupation formula for the semi-martingale local time (see e.g. [22], Corollary 1, p.219). For details, see Lemma 6 in [9], where the case $\sigma>0$ was considered. Because the process of bounded variation is a quadratic pure jump semimartingale (see, e.g. [22], Theorem 26, p.71) then (16) automatically holds.

Theorem 3.2 Suppose that $V^{(q)}$ is sufficiently smooth and that there exists $\left(c_{1}^{*}, c_{2}^{*}\right) \in$ $C^{*}$ such that

$$
V^{(q)^{\prime}}(x) \leq V^{(q)^{\prime}}(y) \text { for all } c_{2}^{*} \leq x \leq y .
$$

Then, the strategy $\pi_{c_{1}^{*}, c_{2}^{*}}$ is an optimal strategy for the impulse control problem.

Proof From Lemma 3.1, one can see that it is sufficient to prove that (13) holds. At first, from the proof of Lemma 3.3, we obtain that for $x<c_{2}^{*},(\Gamma-q) v_{c_{1}^{*}, c_{2}^{*}}^{\kappa^{r}}=0$. In contrast, if $x>c_{2}^{*}$, we get that $(\Gamma-q) v_{c_{1}^{*}, c_{2}^{*}}^{\kappa^{r}} \leq 0$. This follows from the Proposition 3.3 which gives that $v_{c_{1}^{*}, c_{2}^{*}}^{\kappa^{r}}=v_{c_{2}^{*}}^{\kappa^{r}}$, where $v_{c_{2}^{*}}^{\kappa^{r}}$ is the value of the barrier strategy at level $c_{2}^{*}$ in the de Finetti problem and $\lim _{y \uparrow x}(\Gamma-q)\left(v_{c_{2}^{*}}^{\kappa^{r}}-v_{x}^{\kappa^{r}}(y)\right) \leq 0$ for $x>c_{2}^{*}$. The above inequality can be proved using ideas from [9][Theorem 2] together with (17). 


\section{Examples}

In this part, we will present the results concerning the numerical calculations of the optimal impulse policy $\left(c_{1}^{*}, c_{2}^{*}\right)$. From Proposition 3.1, we know that when $C^{*}$ is not an empty set, then $\left(c_{1}^{*}, c_{2}^{*}\right)$ needs to satisfy one of the possibilities listed there. Such an observation will define the way of constructing numerical calculations. However, to even start the computations, one needs to know how to calculate the Parisian refracted scale function. Therefore, we will find an analytical representation for $w^{(q)}$ and $V^{(q)}$ for the linear Brownian motion and for the Crámer-Lundberg process with the exponential claims. Moreover, we will prove that for these two processes, there is a unique $\left(c_{1}, c_{2}\right)$ policy which is optimal for the impulse control problem.

\subsection{Linear Brownian Motion}

Let us assume that process $X$ is a linear Brownian motion which can be represented as $X_{t}=\mu t+\sigma B_{t}$, where $\mu \in \mathbb{R}$ and $\sigma>0$. Fix $q>0$ and $\delta>0$. In such case (see, e.g., [24]) $W^{(q)}(x)=\frac{2}{\sigma^{2} \rho}\left(e^{\rho_{2} x}-e^{-\rho_{1} x}\right)$, where $\rho_{1}=\frac{\sqrt{\mu^{2}+2 q \sigma^{2}}+\mu}{\sigma^{2}}$, $\rho_{2}=\frac{\sqrt{\mu^{2}+2 q \sigma^{2}}-\mu}{\sigma^{2}}, \rho=\rho_{1}+\rho_{2}=\frac{2 \sqrt{\mu^{2}+2 q \sigma^{2}}}{\sigma^{2}}$. For process $Y$, we will use $Y$ superscript for the respective parameters. Our first step is to present the formula for $w^{(q)}$.

Proposition 4.1 For the linear Brownian motion, the function $w^{(q)}$ is of the following form

$$
w^{(q)}(x ;-z)=\frac{\sigma^{2}}{2} W^{(q) \prime}(z) \mathbb{W}^{(q)}(x)+\frac{W^{(q)}(z)}{\rho^{Y}}\left(\rho_{1}^{Y} e^{\rho_{2}^{Y} x}+\rho_{2}^{Y} e^{-\rho_{1}^{Y} x}\right) .
$$

Proof The proof contains simple calculations, which involve the following relations between parameters of $W^{(q)}$ and $\mathbb{W}^{(q)}$

$$
\frac{\rho_{2}}{\rho_{2}-\rho_{2}^{Y}}-\frac{\rho_{2}}{\rho_{1}^{Y}+\rho_{2}}=-\frac{\sigma^{2} \rho^{Y}}{2 \delta}, \frac{\rho_{1}}{\rho_{2}^{Y}+\rho_{1}}+\frac{\rho_{1}}{\rho_{1}^{Y}-\rho_{1}}=-\frac{\sigma^{2} \rho^{Y}}{2 \delta} .
$$

Now, we will consider the formula for the function $V^{(q)}$.

Proposition 4.2 In the linear Brownian motion setting function, $V^{(q)}$ is of the following form. For $x \geq 0$

$$
\begin{aligned}
V^{(q)}(x)= & \frac{\sigma^{2}}{2} \mathbb{W}^{(q)}(x)\left[\frac{2}{\sqrt{2 \pi \sigma^{2} r}} e^{\frac{-r \mu^{2}}{2 \sigma^{2}}}+\rho_{2} e^{q r}-\rho e^{q r} \Phi\left(\frac{-r \sqrt{\mu^{2}+2 q \sigma^{2}}}{\sigma \sqrt{r}}\right)\right] \\
& +\frac{e^{q r}}{\rho^{Y}}\left(\rho_{1}^{Y} e^{\rho_{2}^{Y} x}+\rho_{2}^{Y} e^{-\rho_{1}^{Y} x}\right),
\end{aligned}
$$


for $x<0$

$$
V^{(q)}(x)=e^{q r}\left(e^{\rho_{2} x} \Phi\left(\frac{x+r \sqrt{\mu^{2}+2 q \sigma^{2}}}{\sigma \sqrt{r}}\right)+e^{-\rho_{1} x} \Phi\left(\frac{x-r \sqrt{\mu^{2}+2 q \sigma^{2}}}{\sigma \sqrt{r}}\right)\right),
$$

where $\Phi$ is the cumulative distribution function of the standard normal variable.

Proof We will separate our proof into two parts. Assume that $x \geq 0$. Using formula of the $w^{(q)}$ from the last proposition and Eq. (6), one can get

$$
V^{(q)}(x)=\frac{\sigma^{2}}{2} \mathbb{W}^{(q)}(x) \int_{0}^{\infty} W^{(q) \prime}(z) \frac{z}{r} \mathbb{P}\left(X_{r} \in d z\right)+\frac{e^{q r}}{\rho^{Y}}\left(\rho_{1}^{Y} e^{\rho_{2}^{Y} x}+\rho_{2}^{Y} e^{-\rho_{1}^{Y} x}\right) .
$$

Hence, one needs to calculate integral $\int_{0}^{\infty} W^{(q) \prime}(z) \frac{z}{r} \mathbb{P}\left(X_{r} \in d z\right)$. This simply, but long, calculation is sufficient to end the proof of this part. Now, fix $x<0$. Then $V^{(q)}(x)=\int_{-x}^{\infty} \frac{2}{\sigma^{2} \rho}\left(e^{\rho_{2}(x+z)}-e^{-\rho_{1}(x+z)}\right) \frac{z}{r} \frac{1}{\sqrt{2 \pi \sigma^{2} r}} e^{\frac{-(z-\mu r)^{2}}{2 \sigma^{2} r}} d z$. Therefore, again after some calculations, one can obtain also this part.

Proposition 4.3 For any $q>0$ and $z>0$, there exists a constant $a_{R}^{*} \geq 0$ such that the function $w^{(q)^{\prime}}(x ;-z)$ is decreasing on $] 0, a_{R}^{*}[$ and is increasing on $] a_{R}^{*}, \infty[$. This also implies the same for $V^{(q) \prime}(x)$.

Proof To prove the thesis, we will examine the second derivative with respect to $x$ of $w^{(q)^{\prime}}(x ;-z)$. Indeed, using Proposition 4.1 and the formula for the scale function $\mathbb{W}^{(q)}$, one can get

$$
\begin{aligned}
w^{(q)^{\prime \prime}}(x ;-z)= & \frac{\left(\rho_{2}^{Y}\right)^{2}}{\rho^{Y}} e^{\rho_{2}^{Y} x}\left(W^{(q) \prime}(z)+\rho_{1}^{Y} W^{(q)}(z)\right) \\
& -\frac{\left(\rho_{1}^{Y}\right)^{2}}{\rho^{Y}} e^{-\rho_{1}^{Y} x}\left(W^{(q) \prime}(z)-\rho_{2}^{Y} W^{(q)}(z)\right) \\
= & \frac{\left(\rho_{2}^{Y}\right)^{2}}{\rho^{Y}} e^{\rho_{2}^{Y} x} A-\frac{\left(\rho_{1}^{Y}\right)^{2}}{\rho^{Y}} e^{-\rho_{1}^{Y} x} B,
\end{aligned}
$$

where $\rho_{1}^{Y}, \rho_{2}^{Y}>0$. The constant $A$ is strictly positive because the scale function $W^{(q)}$ is increasing and strictly positive on the whole positive half-line. Now, if $B<0$, then function $w^{(q)^{\prime \prime}}(x ;-z)$ is positive for all $x, z>0$ and hence $a_{R}^{*}=0$. If $B>0$, then $w^{(q)^{\prime \prime}}(x ;-z)$ is an increasing and unbounded function of $x$ as a sum of two increasing exponential functions. This completes the proof for $w^{(q)}$. For $V^{(q) \prime}(x)$, we get the result directly from its definition.

Theorem 4.1 For the linear Brownian motion model, there is a unique $\left(c_{1} ; c_{2}\right)$ policy which is optimal for the impulse control problem.

Proof The thesis of the theorem follows directly from the above Proposition together with Lemma 3.2. For more details, see also Section 4 in [9]. 


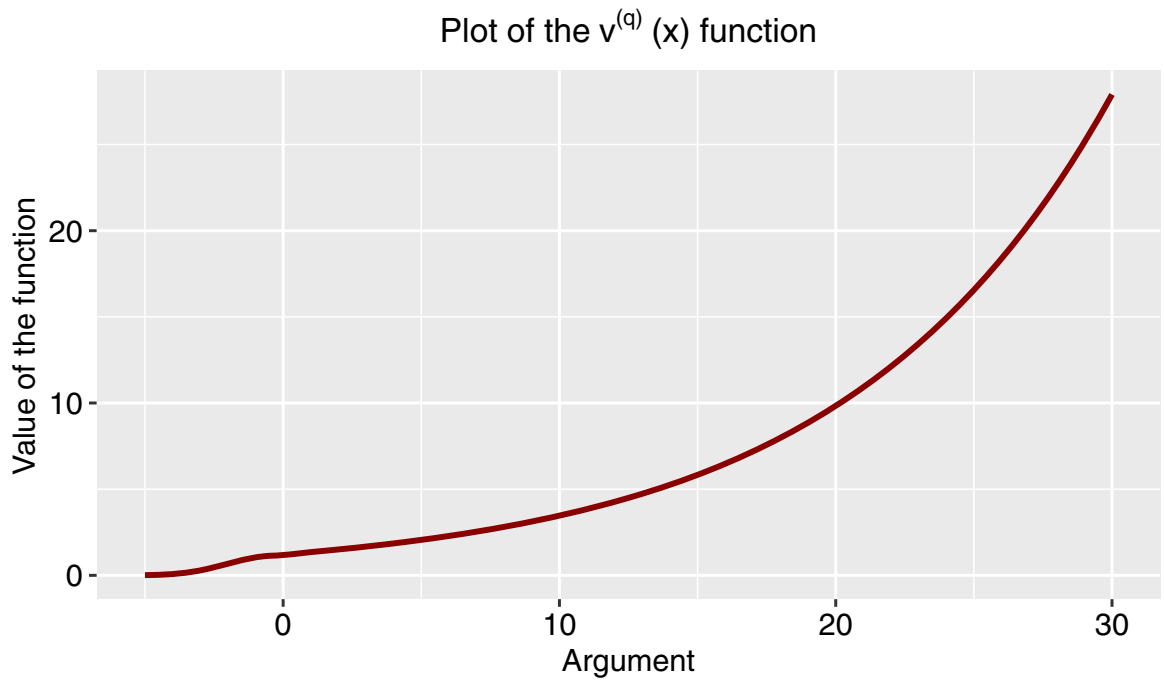

Fig. 1 Plot of the $V^{(q)}$ function for the linear Brownian motion

Now, we will begin numerical examples of $\left(c_{1}^{*}, c_{2}^{*}\right)$ pairs with the respect to three parameters $\beta, r$ and $\delta$. Let us start with the parameter $\beta$ and consider the following parameters $\mu=0.5, \sigma=0.75, r=3, \delta=0.05, q=0.05$. Note that, from Fig. 1 , that the shape of this function is similar as for the classic scale function for linear Brownian motion. In Fig. 2, we consider two plots. On the first plot, we show optimal points $\left(c_{1}^{*}, c_{2}^{*}\right)$ imposed on the graph of $V^{(q) \prime}$. On the second plot, we show $\left(c_{1}^{*}, c_{2}^{*}\right)$ points alone. Both plots are drawn with respect to a varying parameter of $\beta \in[0,1]$. Note that in the second plot, one can see that $c_{1}^{*}$ 's are below point $a_{R}^{*}, c_{2}^{*}$ 's one can find above this level and for fixed $\beta$ pair $\left(c_{1}^{*}, c_{2}^{*}\right)$ is of the same color. One can see that for $\beta$ big enough optimal pair is not belonging to the set $\mathcal{B}$ from the Proposition 3.2. One can think that if the cost of transactions is too restrictive, then optimal behavior is to pay everything that we can, however, $c_{2}^{*}$ must be big enough to have some profit on dividend payment. Moreover, one can be interested in the sensitivity of optimal points with respect to other parameters. Thus, now we will consider parameter $r \in] 0,3]$. We will stay with the same setting of the parameters as before, except $r$.

In Fig. 3 one can see two cases, one for $\beta=0.05$ and second for relative high cost of transaction, namely $\beta=0.45$ Again, for fixed level $r,\left(c_{1}^{*}, c_{2}^{*}\right)$ is of the same color as $r$, where $c_{1}^{*}$ is on bottom curve and $c_{2}^{*}$ is on top one. In both cases, one can see that if we increase parameter $r$ then $\left(c_{1}^{*}, c_{2}^{*}\right)$ will be decreasing in both coordinates. The explanation is quite simple, namely for more significant $r$ process is more safety in terms of possible ruin. Thus, one can lower both barriers to have more often dividends payments. At the end of this part, we will consider sensitivity with respect to parameter $\delta \in] 0,0.25[$. We will consider the following choice of parameters $\mu=0.5, \sigma=0.75$, $r=3, q=0.05$. From Fig. 4, one can, again, observe that changing parameter $\beta$ leads to bigger distance between $c_{1}^{*}$ and $c_{2}^{*}$. Moreover, increasing $\delta$ implies lowering both levels of $c_{1}^{*}$ and $c_{2}^{*}$. This time it is because with increasing of $\delta$, we decrease overall drift of the process. Thus, it is harder to reach higher values. 


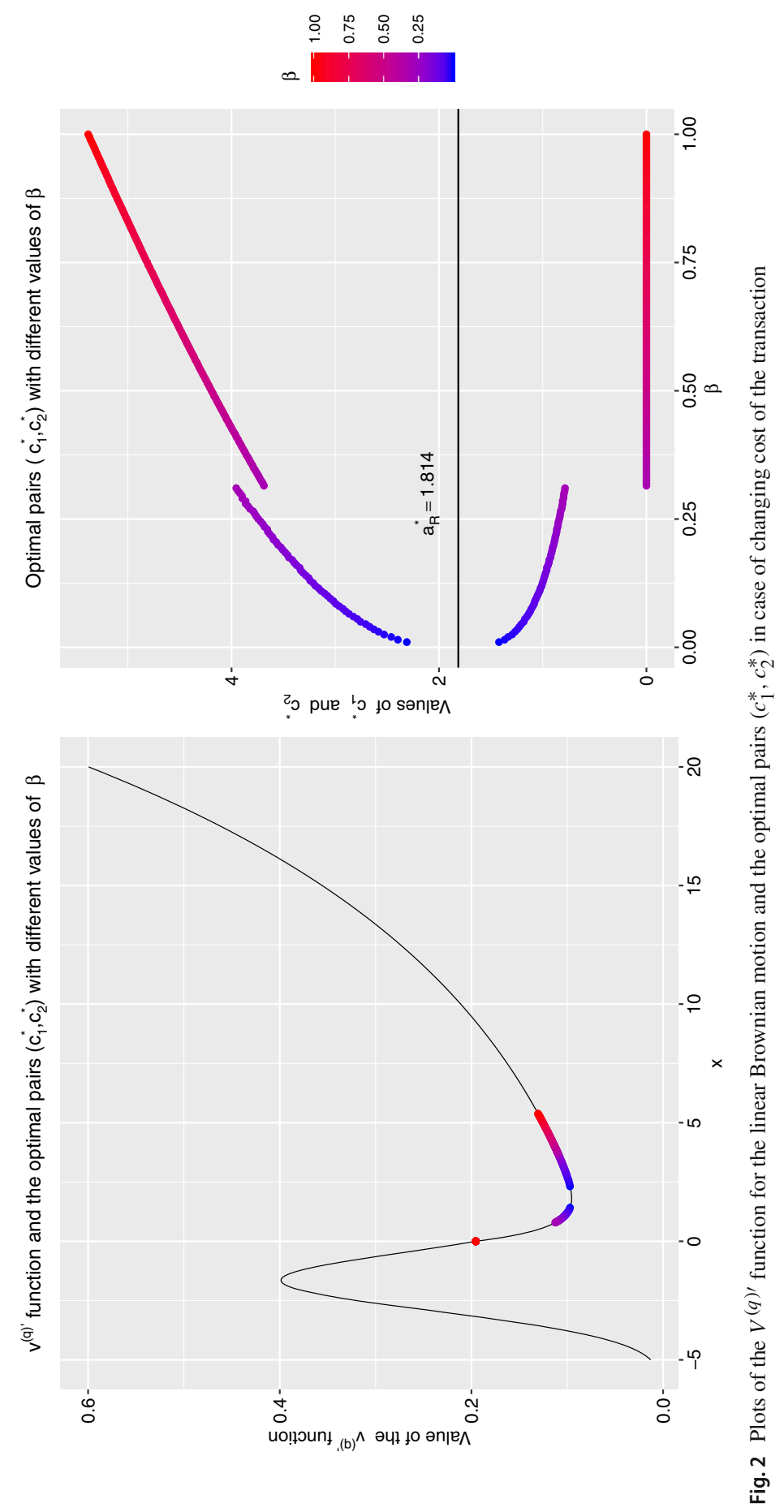



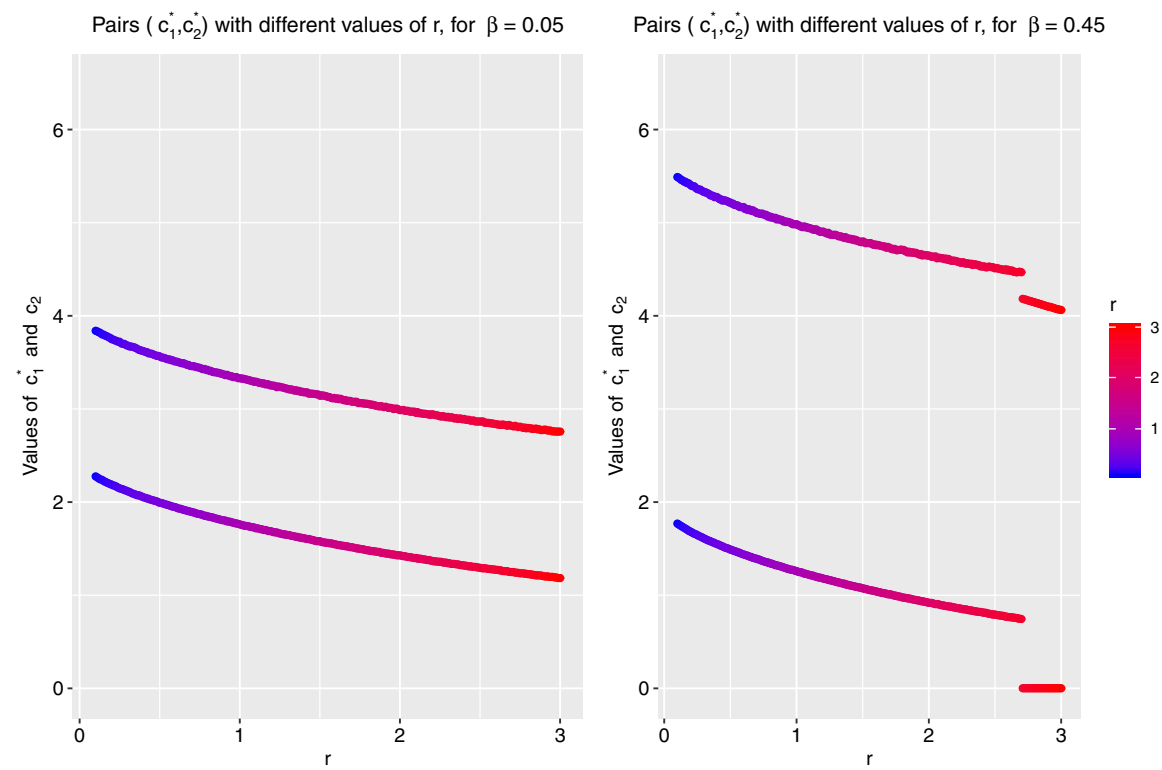

Fig. 3 Plots of the optimal pairs $\left(c_{1}^{*}, c_{2}^{*}\right)$ in case of changing a parameter $r$ for two different values of the parameter $\beta$
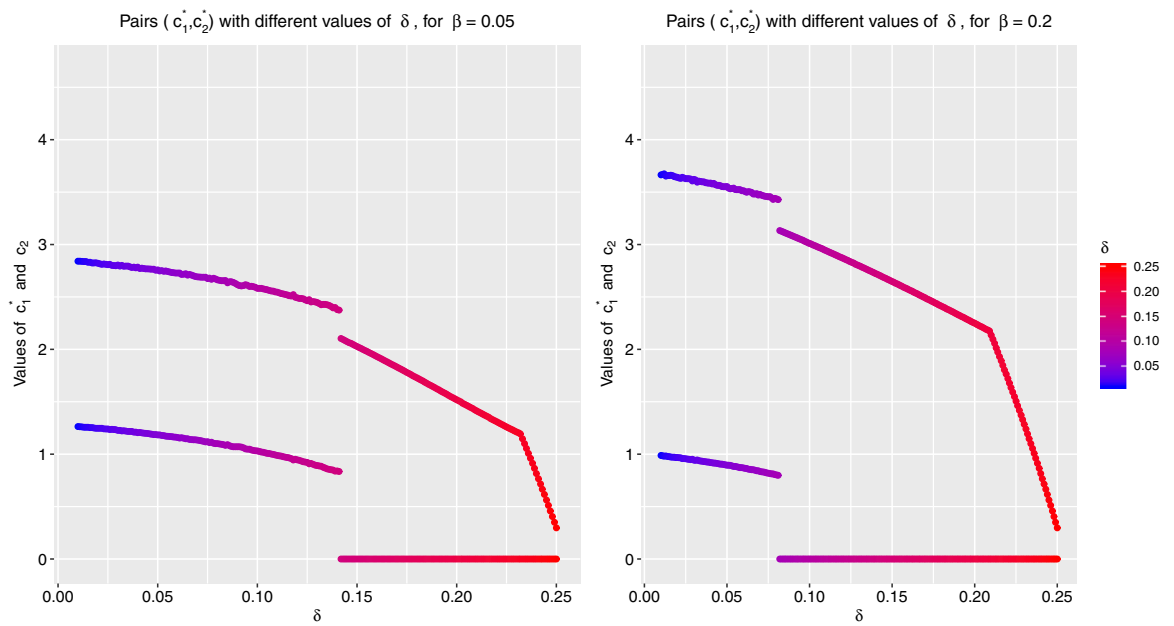

Fig. 4 Plots of the optimal pairs $\left(c_{1}^{*}, c_{2}^{*}\right)$ in case of changing a parameter $\delta$ for two different values of the parameter $\beta$

\subsection{Cramér-Lundberg Process}

In the second example, we will consider the Cramér-Lundberg process $X_{t}=p t-\sum_{i=1}^{N_{t}} U_{i}$, where $p>0,\left\{U_{i}\right\}_{i=1}^{\infty}$ is an $i . i . d$. sequence of exponential random variables with the parameter $\mu>0$, and $\left\{N_{t}\right\}_{t \geq 0}$ is a homogeneous Poisson 
process with the intensity $\lambda>0$. We also assume that the Poisson process and the exponential random variables are mutually independent.

For this process, the scale function is of the following form (see, e.g. [24]) $W^{(q)}(x)=\frac{1}{p}\left(A^{+} e^{q^{+} x}-A^{-} e^{q^{-} x}\right)$, with $q^{ \pm}=\frac{q+\lambda-\mu p \pm \sqrt{(q+\lambda-\mu p)^{2}+4 p q \mu}}{2 p}$ and $A^{ \pm}=\frac{\mu+q^{ \pm}}{q^{+}-q^{-}}$. The respective parameters for the process $Y$ we will denote using superscript $Y$.

Proposition 4.4 For $z>0$, we have that

$$
\begin{aligned}
w^{(q)}(x ;-z)= & (p-\delta) \mathbb{W}^{(q)}(x) W^{(q)}(z)-\frac{1}{\mu \lambda}\left[(q+\lambda) W^{(q)}(z)-p W^{(q) \prime}(z)\right] \\
& \cdot\left[(q+\lambda) \mathbb{W}^{(q)}(x)-(p-\delta) \mathbb{W}^{(q)^{\prime}}(x)\right] .
\end{aligned}
$$

Proof To obtain such a representation, we had to use the following relations between the parameters of scale functions

$$
\begin{array}{ll}
\frac{A_{Y}^{+} q^{+}}{q^{+}-q_{Y}^{+}}-\frac{A_{Y}^{-} q^{+}}{q^{+}-q_{Y}^{-}}=-\frac{p-\delta}{\delta}, & \frac{A_{Y}^{-} q^{-}}{q^{-}-q_{Y}^{-}}-\frac{A_{Y}^{+} q^{-}}{q^{-}-q_{Y}^{+}}=\frac{p-\delta}{\delta}, \\
\frac{q^{+}}{q^{+}-q_{Y}^{+}}=-\frac{p-\delta}{\delta} \cdot \frac{q^{+}-q_{Y}^{-}}{q^{+}+\mu}, & \frac{q^{-}}{q^{-}-q_{Y}^{+}}=-\frac{p-\delta}{\delta} \cdot \frac{q^{-}-q_{Y}^{-}}{q^{-}+\mu} .
\end{array}
$$

We will obtain the formula for a Parisian refracted scale function, which will be divided into three parts.

Proposition 4.5 In the Crámer-Lundberg setting, the function $V^{(q)}$ is of the following form. For $x>0$,

$$
\begin{aligned}
& V^{(q)}(x)= e^{q r}(p-\delta) \mathbb{W}^{(q)}(x)-\frac{1}{\mu \lambda}\left[(q+\lambda) \mathbb{W}^{(q)}(x)-(p-\delta) \mathbb{W}^{(q)^{\prime}}(x)\right] \\
& \cdot\left[(q+\lambda) e^{q r}-p C\right], \\
& C:=e^{-\lambda r}\left[p W^{(q) \prime}(p r)+C^{+}-C^{-}+\frac{e^{-\mu p r}}{p r} \sum_{m=1}^{\infty} \frac{\left(p \lambda \mu r^{2}\right)^{m}}{(m-1) ! m !}\right], \\
& C^{ \pm}:=A^{\mp} q^{ \pm} e^{q^{ \pm}} p r \sum_{m=1}^{\infty} \frac{\left.\operatorname{pr}\left(q^{\mp}+\mu\right)\right)^{(m-1)}}{(m-1) ! m !} \gamma\left(m,\left(q^{ \pm}+\mu\right) p r\right)\left[p r\left(q^{ \pm}+1\right)-m\right],
\end{aligned}
$$

where $\gamma(x, a)=\int_{0}^{x} e^{-t} t^{a-1} d t$ is an incomplete gamma function. 
For $x \in[-p r, 0], V^{(q)}(x)=e^{-\lambda r}\left[p W^{(q)}(x+p r)+K^{+}(x)-K^{-}(x)\right]$, with

$$
\begin{aligned}
K^{ \pm}(x):= & A^{\mp} e^{q^{ \pm}(x+p r)} \sum_{m=1}^{\infty} \frac{\left(p r\left(q^{\mp}+\mu\right)\right)^{m-1}}{(m-1) ! m !} \gamma\left(m,(p r+x)\left(q^{ \pm}+\mu\right)\right) \\
& \cdot\left[p r\left(q^{ \pm}+\mu\right)-m\right] .
\end{aligned}
$$

For $x<-p r$, we have $V^{(q)}(x)=0$.

Proof We will divide this proof into the three parts. For $x>0$ we use the formula from the Proposition 4.4 and Eq. (6)

$$
\begin{aligned}
V^{(q)}(x)= & (p-\delta) \mathbb{W}^{(q)}(x) e^{q r}-\frac{1}{\mu \lambda}\left[(q+\lambda) \mathbb{W}^{(q)}(x)-(p-\delta) \mathbb{W}^{(q)^{\prime}}(x)\right] \\
& \cdot\left[(q+\lambda) e^{q r}-p \int_{0}^{\infty} W^{(q)^{\prime}}(z) \frac{z}{r} \mathbb{P}\left(X_{r} \in d z\right)\right] .
\end{aligned}
$$

Let us recall that, one can obtain from [15], the following

$$
\mathbb{P}\left(\sum_{i=1}^{N_{r}} U_{i} \in d y\right)=e^{-\lambda r}\left(\delta_{0}(d y)+e^{-\mu y} \sum_{m=1}^{\infty} \frac{(\mu \lambda r)^{m}}{(m-1) ! m !} y^{m-1} d y\right)
$$

With the use of (19), it turns out that $\int_{0}^{\infty} W^{(q) \prime}(z) \frac{z}{r} \mathbb{P}\left(X_{r} \in d z\right)=C$. Putting all the pieces together, one gets the postulated formula for $V^{(q)}$ for $x>0$.

Now, fix $x \in[-p r, 0]$. In this case, $V^{(q)}(x)=\int_{0}^{\infty} W^{(q)}(x+z) \frac{z}{r} \mathbb{P}\left(X_{r} \in d z\right)$. Random variable $X_{r}$ can achieve at most value $p r$ with the probability one. Moreover, we know that $W^{(q)}(x+z)>0$ iff $x+z>0$. Therefore,

$V^{(q)}(x)=\int_{-x}^{p r} W^{(q)}(x+z) \frac{z}{r} \mathbb{P}\left(X_{r} \in d z\right)$. Using this observation, the rest of the proof involves simple but long calculations; thus, we omit this.

Fix $x<-p r$. As we state in the previous case, when $x<-p r$, then $x+z<0$. Therefore, $W^{(q)}(x+z)=0$ and $V^{(q)}(x)=0$.

Proposition 4.6 Fix $q>0$ and $z>0$, there exists a constant $a_{R}^{*} \geq 0$ such that the function $w^{(q)^{\prime}}(x ;-z)$ is decreasing on $] 0, a_{R}^{*}[$ and is increasing on $] a_{R}^{*}, \infty[$. This also implies the same for $V^{(q) \prime}(x)$.

Proof Firstly, let us note that $\frac{q+\lambda}{p-\delta}-q_{Y}^{+}=q_{Y}^{-}+\mu$ and $\frac{q+\lambda}{p}-q^{+}=q^{-}+\mu$. From this observation and Proposition 4.4, one can obtain

$$
\begin{aligned}
& (q+\lambda) \mathbb{W}^{(q)}(x)-(p-\delta) \mathbb{W}^{(q)^{\prime}}(x)=\frac{\mu \lambda\left[e^{q_{Y}^{+} x}-e^{q_{Y}^{-} x}\right]}{(p-\delta)\left(q_{Y}^{+}-q_{Y}^{-}\right)}, \\
& (q+\lambda) W^{(q)}(z)-p W^{(q) \prime}(z)=\frac{\mu \lambda\left[e^{q^{+} z}-e^{q^{-} z}\right]}{p\left(q^{+}-q^{-}\right)} .
\end{aligned}
$$


So, $w^{(q)}(x ;-z)=(p-\delta) \mathbb{W}^{(q)}(x) W^{(q)}(z)-\frac{\mu \lambda\left[e^{q^{+} z}-e^{q^{-} z}\right]\left[e^{q_{Y}^{+} x}-e^{q_{Y}^{-} x}\right]}{p(p-\delta)\left(q^{+}-q^{-}\right)\left(q_{Y}^{+}-q_{Y}^{-}\right)}$. Thus, one can also get the more explicit form $w^{(q)}(x ;-z)=D^{+} e^{q_{Y}^{+} x}-D^{-} e^{q_{Y}^{-} x}$, where

$$
D^{ \pm}=A_{Y}^{ \pm} W^{(q)}(z)-\frac{\mu \lambda\left(e^{q^{+} z}-e^{q^{-} z}\right)}{p(p-\delta)\left(q^{+}-q^{-}\right)\left(q_{Y}^{+}-q_{Y}^{-}\right)} .
$$

Next, $q_{Y}^{+}>0, q_{Y}^{-}<0$ and $\lim _{x \rightarrow+\infty} e^{q_{Y}^{+} x}=+\infty, \lim _{x \rightarrow+\infty} e^{q_{Y}^{-} x}=0$. Then, from $\lim _{x \rightarrow+\infty} w^{(q)}(x ;-z)=+\infty$, one can get that $D^{+}>0$. Moreover, we are interested in the sign of $w^{(q)^{\prime \prime}}(x ;-z)=D^{+}\left(q_{Y}^{+}\right)^{2} e^{q_{Y}^{+} x}-D^{-}\left(q_{Y}^{-}\right)^{2} e^{q_{Y}^{-} x}$. If $D^{-}<0$, then $w^{(q)^{\prime \prime}}(x ;-z)$ is positive on the whole positive half-line. In such a case, $a_{R}^{*}=0$. If $D^{-}>0$, then one can see that $w^{(q)^{\prime \prime}}(x ;-z)$ is an increasing and unbounded function. This ends the proof.

Theorem 4.2 For the Cramér-Lundberg model, there is a unique $\left(c_{1} ; c_{2}\right)$ policy which is optimal for the impulse control problem.

Proof The thesis of the theorem follows directly from the above Proposition together with Lemma 3.2. For more details, see also Section 4 in [9].

Using the above results, one can plot the picture of the $V^{(q)}$ and $V^{(q) \prime}$ for this process. Namely, let us set $p=3, \lambda=2, \mu=1, r=2, q=0.05, \delta=0.25$. Note that we set such parameters that $p>\frac{\lambda}{\mu}$. Moreover, we know that $V^{(q)}(x)=0$ if $x<-p r$; therefore, we will consider $\mathrm{x} \geq-p r$.

From Fig. 5, as in the linear Brownian motion setting, one can also see a similar shape of the Parisian scale function with the shape of a classical scale function. However, even if this is not directly clear from Fig. 5, $V^{(q)}$ is not a continuous function at $x=-p r$.

Now, we will proceed to sensitive analyse of three parameters $\beta, r$ and $\delta$, as it was a case for linear Brownian motion. Let us start with the parameter $\beta \in] 0,1.5]$ From Fig. 6, one can see that $V^{(q)^{\prime}}$ is not a continuous function at point 0 . Moreover, from the right plot, one can see that increasing parameter $\beta$ causes on the increasing distance between $c_{1}^{*}$ and $c_{2}^{*}$. Furthermore, again one can see that for big enough $\beta$ optimal pair $\left(c_{1}^{*}, c_{2}^{*}\right)$ does not belong to the set $\mathcal{B}$. Let us proceed to Fig. 7 , where one can see sensitivity for the parameter $r \in] 0,3]$. From this picture, one can see that if one would increase the parameter $r$ then optimal points $\left(c_{1}^{*}, c_{2}^{*}\right)$ are both lowering their levels. This is due to being safer from ruin, and thus one can stick closer to level 0. At last, but not least let us consider sensitivity for the parameter $\delta \in[0.1,0.5]$. In Fig. 8, one can see that with increasing the parameter $\delta$ optimal points $\left(c_{1}^{*}, c_{2}^{*}\right)$ are decreasing in both coordinates. This is consistent with the previous conclusions on $\delta$.

\section{Comments}

In this article, we solved the problem of optimal dividend payments for refracted spectrally negative Levy processes. In our model, we proposed Parisian ruin time 


\section{Plot of the $v^{(q)}(x)$ function}

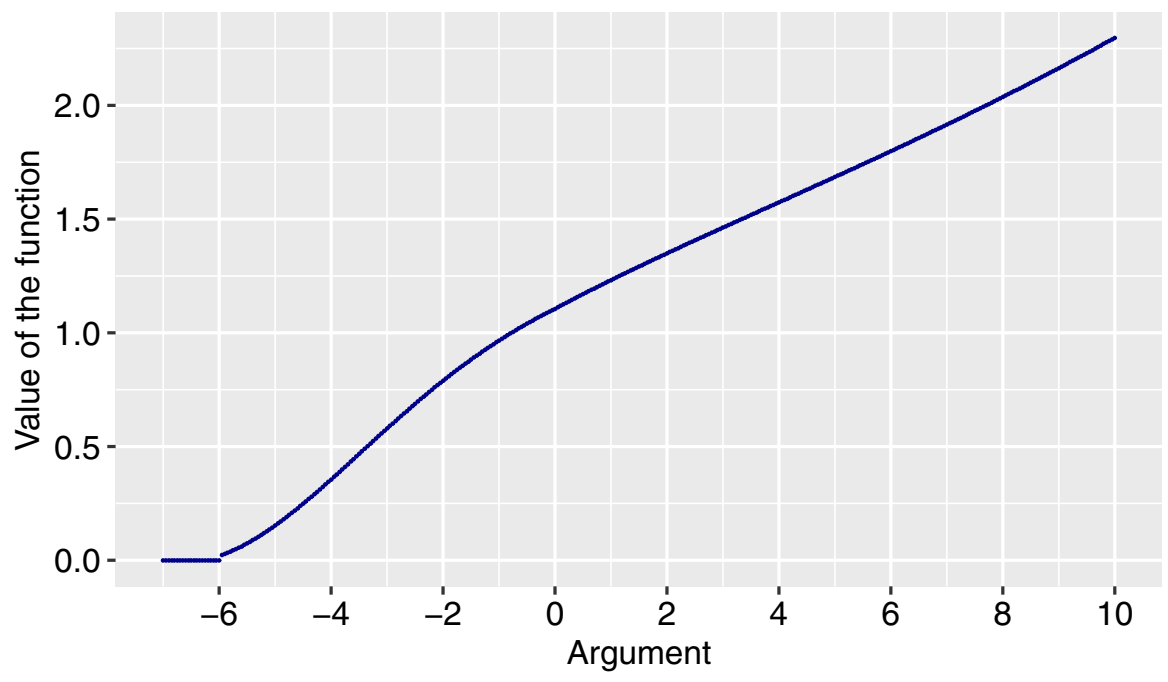

Fig. 5 Plot of the $V^{(q)}$ for the Cramér-Lundberg process
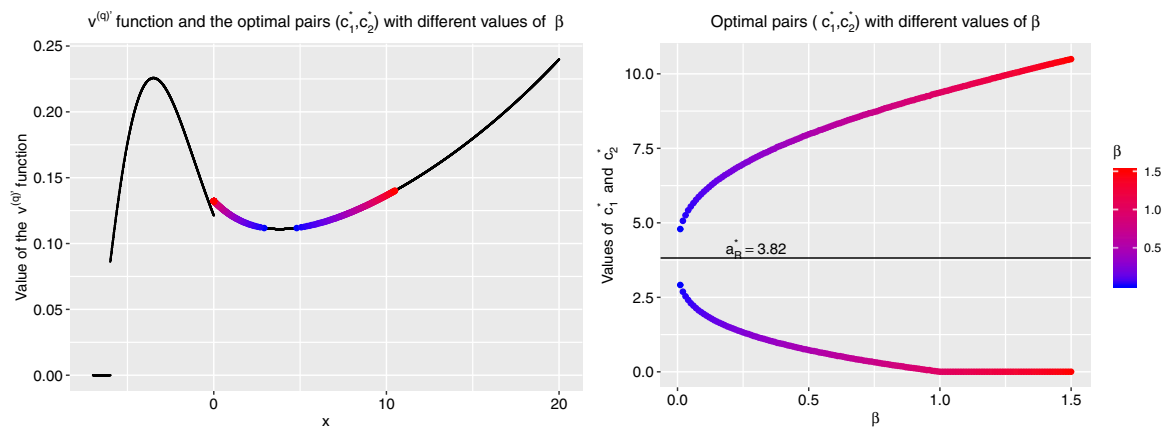

Fig. 6 Plot of the $V^{(q)^{\prime}}$ for the Cramér-Lundberg process and the optimal pairs $\left(c_{1}^{*}, c_{2}^{*}\right)$ in the case of changing the cost of the transaction

as a definition of ruin, and we assumed that every dividend payment is associated with fixed transaction cost. Even though this setting was motivated by being close to reality, and we firmly believe that with success, there is a big room for future developments. Namely, we interpret the refracted model as a cash injection done by shareholders and dividend as a payout for them. However, we did not connect these two interpretations. It would be worthy to see some penalty function for being in the red zone which will be associated with the value function of dividend payments. In such case finding optimal pair $\left(c_{1}^{*}, c_{2}^{*}\right)$ would consider not only the risk of ruin but also the risk of staying in the red zone. One possibility is to remove cash injected through the refracted model from the present value of value function. Another idea could be to have more sophisticated discounting structure, which would take care of how long we stayed in the red zone. Without these improvements, one can see, for 

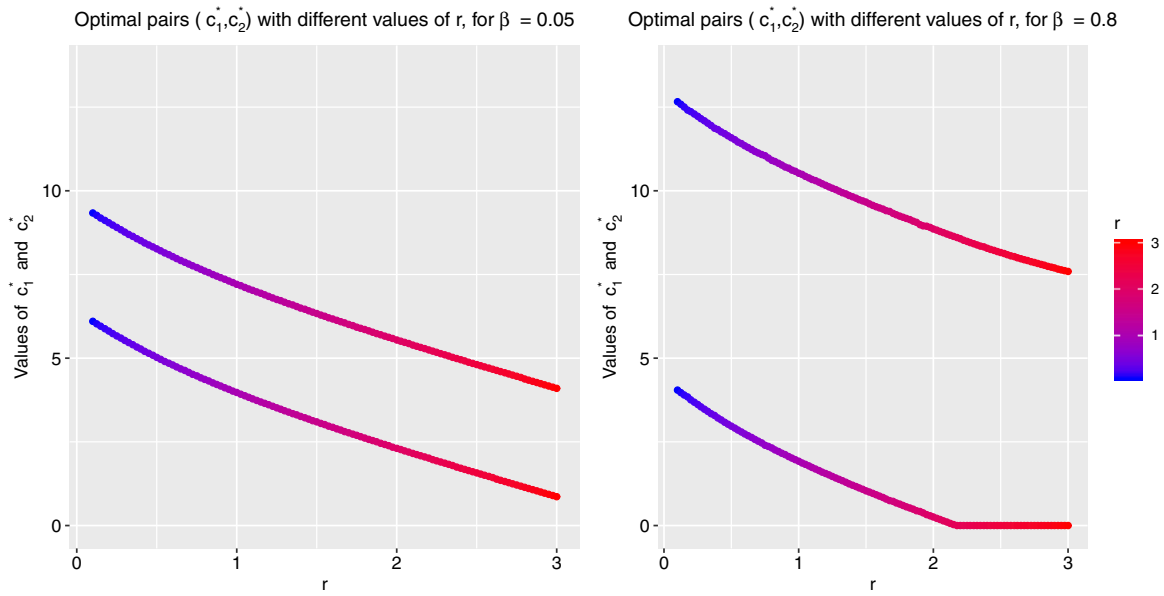

Fig. 7 Plot of the optimal pairs $\left(c_{1}^{*}, c_{2}^{*}\right)$ in case of changing a parameter $r$ for two different values of the parameter $\beta$
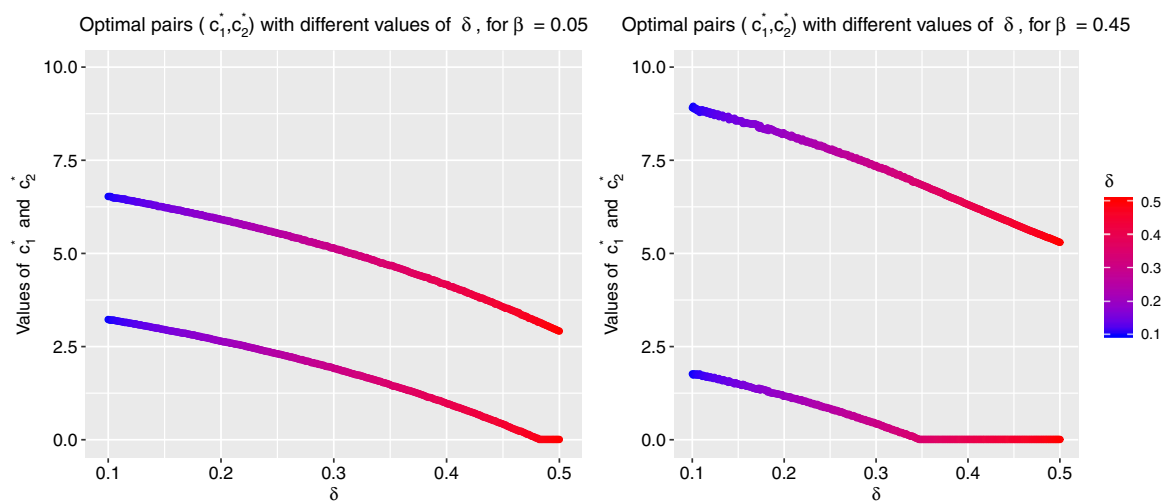

Fig. 8 Plot of the optimal pairs $\left(c_{1}^{*}, c_{2}^{*}\right)$ in case of changing a parameter $\delta$ for two different values of the parameter $\beta$

example through a numerical section, that increasing parameters $\delta$ and $r$ will lead to the same effect of decreasing $\left(c_{1}^{*}, c_{2}^{*}\right)$ in both coordinates, which could be misleading. Namely, increasing the value of parameter $r$ can be seen as a decreasing probability of ruin, however increasing $\delta$ means that we lower our potential drift of the process. Furthermore, one can see that there can be done a lot more in the case of numerical computation. In our article, we focus on giving some intuitions behind the model. However, one could be interested, for example, in some semi-explicit results on the behavior of optimal points with respect to model parameters.

Acknowledgements We would like to thanks an anonymous referee and an editor for their valuable suggestions which improved the quality of the article, especially for giving an idea of the sensitivity in the numerical section. I. Czarna is partially supported by the National Science Centre Grant No. 2015/19/D/ST1/01182. A. Kaszubowski is partially supported by the National Science Centre Grant No. 2015/17/B/ST1/01102. 
Open Access This article is licensed under a Creative Commons Attribution 4.0 International License, which permits use, sharing, adaptation, distribution and reproduction in any medium or format, as long as you give appropriate credit to the original author(s) and the source, provide a link to the Creative Commons licence, and indicate if changes were made. The images or other third party material in this article are included in the article's Creative Commons licence, unless indicated otherwise in a credit line to the material. If material is not included in the article's Creative Commons licence and your intended use is not permitted by statutory regulation or exceeds the permitted use, you will need to obtain permission directly from the copyright holder. To view a copy of this licence, visit http://creativecommons.org/licenses/by/4.0/.

\section{References}

1. Chesney, M., Jeanblanc-Picqué, M., Yor, M.: Brownian excursions and Parisian barrier options. Adv. Appl. Probab. 29, 165-184 (1997)

2. Czarna, I., Palmowski, Z.: Ruin probability with Parisian delay for a spectrally negative Lévy risk proces. Appl. Probab. 48, 984-1002 (2011)

3. Kyprianou, A.E., Loeffen, R.L.: Refracted Lévy processes. Annales de l'Institut Henri Poincaré Probabilités et Statistiques 46(1), 24-44 (2010)

4. de Finetti, B.: Su unímpostazion alternativa dell teoria collecttiva del rischio. Trans. XVth Int. Congr. Actuar. 2, 433-443 (1957)

5. Gerber, H.U., Shiu, E.S.W.: Optimal dividends: analysis with Brownian motion. N. Am. Actuar. J. 8, 1-20 (2004)

6. Gerber, H.U., Shiu, E.S.W.: On optimal dividend strategies in the compound poisson model. N. Am. Actuar. J. 10, 76-93 (2006)

7. Jeanblanc, M., Shiryaev, A.N.: Optimization of the flow of dividends. Russ. Math. Surv. 50, 257-277 (1995)

8. Avram, F., Palmowski, Z., Pistorius, M.R.: On the optimal dividend problem for a spectrally negative Lévy process. Ann. Appl. Probab. 17, 156-180 (2007)

9. Loeffen, R.L.: An optimal dividends problem with transaction costs for spectrally negative Lévy processes. Ann. Appl. Probab. 18(5), 1669-1680 (2008)

10. Bertoin, J.: Lévy Processes. In: Cambridge Tracts in Mathematics. Cambridge University Press, Cambridge (1996)

11. Kyprianou, A.E.: Introductory Lectures on Fluctuations of Lévy Processes with Applications. Springer, Berlin (2006)

12. Lkabous, M.A., Czarna, I., Renaud, J.-F.: Parisian ruin for a refracted Lévy process. Insur. Math. Econ. 47, 153-165 (2017)

13. Hubalek, F., Kyprianou, E.: Old and new examples of scale functions for spectrally negative Lévy processes. In: Dalang, R., Dozzi, M., Russo, F. (eds.) Seminar on Stochastic Analysis, Random Fields and Applications VI. Progress in Probability, vol. 63, pp. 119-145. Springer, Basel (2011)

14. Kuznetsov, A., Kyprianou, A.E., Rivero, V.: The Theory of Scale Functions for Spectrally Negative Lévy Processes. Lévy Matters II. Lecture Notes in Mathematics, Vol. 2061. Springer, Berlin (2012)

15. Loeffen, R.L., Czarna, I., Palmowski, Z.: Parisian ruin probability for spectrally negative Lévy processes. Bernoulli 19(2), 599-609 (2013)

16. Czarna, I., Pérez, J.-L., Rolski, T., Yamazaki, K.: Fluctuation theory for level-dependent Lévy risk processes. Stoch. Process. Their Appl. 129(12), 5406-5449 (2019). https://doi.org/10.1016/j.spa.2019. 03.006

17. Noba, K., Yano, K.: Generalized refracted Lévy process and its application to exit problem. Stoch. Process. Their Appl. 129(5), 1697-1725 (2019)

18. Khoshnevisan, D., Schilling, R.: From Lévy-Type Processes to Parabolic SPDEs. Advanced Courses in Mathematics. CRM Barcelona, Birkhäuser Basel (2016)

19. Schilling, R.L.: Growth and Hölder conditions for the sample paths of a Feller process. Probab. Theor. Relat. Fields 112, 565-611 (1998)

20. Schnurr, A.: On the semimartingale nature of Feller processes with killing. Stoch. Proc. Appl. 122, 2758-2780 (2012)

21. Bouleau, N., Yor, M.: Sur la variation quadratique des temps locaux de certaines semimartingales. C. R. Acad. Sci. Paris 292, 491-494 (1981) 
22. Protter P.: Stochastic Integration and Differential Equations, 2nd ed., version 2.1. Springer, Berlin (2005)

23. Kyprianou, A.E., Rivero, V., Song, R.: Smoothness and convexity of scale functions with applications to de Finetti's control problem. J. Theor. Probab. 23, 547 (2010)

24. Czarna, I.: Parisian ruin probability with a lower ultimate bankrupt barrier. Scand. Actuar. J. 2016(4), 319-337 (2016)

Publisher's Note Springer Nature remains neutral with regard to jurisdictional claims in published maps and institutional affiliations. 